\title{
Phosphatase PP2C6 negatively regulates phosphorylation status of Pti1b kinase, a regulator of flagellin-triggered immunity in tomato
}

\section{Fabian Giska ${ }^{1}$ and Gregory B. Martin ${ }^{1,2 *}$}

${ }^{1}$ Boyce Thompson Institute for Plant Research, Ithaca, New York 14853, USA; ${ }^{2}$ Section of Plant Pathology and Plant-Microbe Biology, School of Integrative Plant Science, Cornell University, Ithaca, New York 14853, USA

*To whom correspondence should be addressed: Gregory B. Martin, Boyce Thompson Institute for Plant Research, Ithaca, New York 14853.

E-mail: gbm7@cornell.edu; Phone: +1-607-254-1208

Key words: Pti1b, kinase, phosphatase, PP2C6, reactive oxygen species, tomato, plant immunity, phosphorylation

Running title: PP2C6 phosphatase and plant immunity

\begin{abstract}
Abbreviations: ANXR, anxur receptor-like kinase; BAK1, brassinosteroid insensitive 1-associated kinase 1; BIK1, botrytis-induced kinase1; CERK1, chitin elicitor receptor kinase 1; CORE, Cold shock protein receptor; csp, cold shock protein; CWI, cell wall integrity; EGF, epidermal growth factor; flg, flagellin; FLS2, flagellin sensing 2; KAPP, kinase associated protein phosphatase; LRR, leucine-rich repeat; MAMPs, microbe-associated molecular patterns; MAPKs, mitogen-activated protein kinases; NADPH, nicotinamide adenine dinucleotide phosphate, Oxi1, oxidative signal-inducible1; PBL, avrPphB susceptible-like; PP2C, protein phosphatase 2C; PRRs, pattern recognition receptors; PTI, patterntriggered immunity; Ptilb, Pto interactor 1b; RBOHD, respiratory burst oxidase homolog protein D; RLCKs, receptor-like cytoplasmic kinases; ROS, reactive oxygen species; XB15, XA21 binding protein 15.
\end{abstract}


PP2C6 phosphatase and plant immunity

\begin{abstract}
Plant immune responses, including the production of reactive oxygen species (ROS), are triggered when pattern recognition receptors (PRR) become activated upon detection of microbe-associated molecular patterns (MAMPs). Receptor-like cytoplasmic kinases are key components of PRR-dependent signaling pathways. In tomato two such kinases, Ptila and Ptilb, are important positive regulators of the plant immune response. However, it is unknown how these kinases control plant immunity at the molecular level, and how their activity is regulated. To investigate these issues, we used mass spectrometry to search for interactors of Ptilb in Nicotiana benthamiana leaves and identified a protein phosphatase, PP2C6. An in vitro pull-down assay and in vivo split luciferase complementation assay verified this interaction. Ptilb was found to autophosphorylate on threonine-233 and this phosphorylation was abolished in the presence of PP2C6. An arginine-to-cysteine substitution at position 240 in the Arabidopsis MARIS kinase was previously reported to convert it into a constitutive-active form. The analogous substitution in Ptilb made it resistant to PP2C6 phosphatase activity, although it still interacted with PP2C6. Treatment of $N$. benthamiana leaves with the MAMP flg22 induced threonine phosphorylation of Pti1b. Expression of PP2C6, but not a phosphatase-inactive variant of this protein, in N. benthamiana leaves greatly reduced ROS production in response to treatment with MAMPs flg22 or csp22. The results indicate that PP2C6 acts as a negative regulator by dephosphorylating the Ptilb kinase, thereby interfering with its ability to activate plant immune responses.
\end{abstract}


PP2C6 phosphatase and plant immunity

\section{Introduction}

Plants exist in an environment containing large numbers of diverse microorganisms, some of them detrimental to plant health. Consequently, their survival depends on their ability to specifically recognize and respond to pathogenic microbes. This recognition is enabled by the function of plasma membranelocalized pattern recognition receptors (PRRs), which recognize microbe-associated molecular patterns (MAMPs) (1-3). The best understood PRR is FLS2, which perceives the peptide flg22, a part of the flagellin protein, which forms the bacterial flagellum $(4,5)$. Two PRRs that are present only in solanaceous species, including tomato, are FLS3, which recognizes a flagellin-derived peptide, flgII-28, and CORE, which detects a MAMP in the bacterial cold shock protein, csp22 (6,7). The binding of the extracellular part of the receptor to the appropriate ligand activates the cytoplasmic kinase domain of the receptor (8).

Activated PRRs induce a defense response referred to as pattern triggered immunity (PTI). PTI is associated with the generation of reactive oxygen species (ROS), activation of mitogen-activated protein kinases (MAPKs), transcriptional reprogramming, production of antimicrobial molecules, and the reinforcement of the cell wall, among other processes (9-12). Receptor-like cytoplasmic kinases (RLCK) play a key role in linking PRRs to downstream signaling components (13). RLCKs constitute a large family of proteins consisting primarily of a serine-threonine kinase domain but some members also possess EGF, LRR, WD40 and transmembrane domains (13).

The Arabidopsis thaliana genome encodes 179 RLCKs divided into 17 subfamilies: RLCK-II and from RLCK-IV to RLCK-XIX. The majority of RLCKs which are known to have a role in plant immunity belong to sub-family VII. For example, AtBIK1, a member of sub-family VII, is activated upon phosphorylation by FLS2 after its perception of flg22 $(13,14)$. The activated BIK1 induces generation of ROS by phosphorylating the NADPH oxidase, RBOHD $(11,15)$. Similarly, the activated PRR chitin elicitor receptor kinase 1 (CERK1) phosphorylates the RLCK protein PBL27, which then directly activates an immunity-associated MAPK kinase cascade (16). Some RLCKs play a negative regulatory role such as PBL13 whose activation reduces generation of ROS in Arabidopsis and hinders resistance against the bacterial pathogen Pseudomonas syringae pv. tomato (17).

RLCKs also play important roles in regulating plant growth, development and reproduction. One such RLCK is MARIS, which belongs to sub-family VIII. MARIS was identified in a forward genetic screen as a suppressor of the anx1/anx2 mutations (18). This double mutation disrupts cell wall integrity (CWI)signaling and results in a burst of the pollen tube after pollen germination. The MARIS allele identified in the screen contains a mutation that introduces an arginine-to-cysteine substitution at position 240 
PP2C6 phosphatase and plant immunity

(R240C) in the activation loop of the kinase which rescues the pollen tube bursting phenotype of the anx1/anx2 mutant (18). This and other observations suggested that MARIS acts downstream of the ANX1/ANX2 receptors in CWI signaling and that the R240C variant is a constitutively-active form of MARIS (18), although the molecular basis for this constitutive activity is unknown.

Defense responses in plants need to be tightly regulated to prevent damage to plant tissue. Since immune responses are often activated by protein kinases, protein phosphatases are natural candidates as a negative regulators of plant responses to pathogen attack. Indeed a few phosphatases are known to function as regulators of PRRs, immunity-associated RLCKs, and MAPKs. The kinase associated protein phosphatase (KAPP), for example, interacts with FLS2 and KAPP overexpression blocks flg22-dependent signaling (19). The rice PRR XA21 which confers resistance to the bacterial pathogen Xanthomonas oryzae pv. oryzae, interacts with XB15 which belongs to the protein phosphatase 2C (PP2C) family (20). XB15 dephosphorylates the autophosphorylated kinase domain of XA21. Rice plants with a mutation in the $\mathrm{Xb15}$ gene show symptoms of activated defense responses whereas plants overexpressing $X b 15$ are more susceptible to X. o. pv. oryzae (20). In Nicotiana benthamiana, virus-induced gene silencing of a gene encoding a catalytic subunit of protein phosphatase $2 \mathrm{~A}$ results in activation of plant defense responses but the mechanism is not known (21). In Arabidopsis, the PRR co-receptor BAK1 interacts with a phosphatase PP2A holoenzyme. PP2A also negatively controls phosphorylation status of BAK1 thereby interfering with subsequent PTI signaling (22). The RLCK BIK1 is known to interact with phosphatase PP2C38. The phosphatase dephosphorylates BIK1 and impedes its ability to phosphorylate the NADPH oxidase RBOHB causing impaired ROS production and decreased stomatal-mediated immune responses (23). Finally, some MAPK phosphatases are known to negatively regulate aspects of PTI including ROS production $(24,25)$.

In tomato, two highly similar kinases referred as Pti1a (Pto interactor 1a) and Pti1b play a role in patterntriggered immunity against $P$. s. pv. tomato (26). Like MARIS, Pti1a and Ptilb are members of RLCK family VIII and consist primarily of a serine-threonine protein kinase domain (26). We previously generated and characterized tomato plants carrying a hairpin (hp)-Ptil construct which consequently have reduced expression of both Ptila and Ptilb. The hpPtil transgenic plants are impaired in ROS production in response to treatment with MAMPs flg22 or flgII-28, whereas activation of MAPK kinases is unaffected (26). Importantly, the hpPti1 plants are significantly more susceptible to $P$. s. pv. tomato. Ptila was previously shown to intramolecularly autophosphorylate on threonine-233 in the activation loop of the kinase; however, the possible role of this phosphorylation in Ptila-mediated PTI signaling is unknown $(27,28)$. 
PP2C6 phosphatase and plant immunity

Homologs of the Ptil kinases exist in many other plant species. In Arabidopsis it was shown that Pti1-2 and Pti1-4 play a role in oxidative stress $(29,30)$, and the kinase activity of Pti1-2 is induced by flg22 (29). Arabidopsis Pti1-1 and Pti1-2 proteins, but not Pti1-3 or Pti1-4, show strong autophosphorylation activity (29). In cucumber, a Pti1 homolog CsPti1-L is a positive regulator of plant immunity and salt tolerance, and also exhibits autophosphorylation activity (31). In contrast, it was shown in rice that OsPtila acts as a negative regulator of basal defense against X. o. pv. oryzae and Magnaporthe oryzae $(32,33)$. As with the tomato Ptila kinase, threonine-233 of OsPtila undergoes autophosphorylation in vitro and additionally is phosphorylated by the OsOxi1 kinase (33). The authors postulate that the negative regulatory function of OsPtila might be abolished by OsOxi1-dependent phosphorylation of OsPti1a on threonine 233 (33). An OsPti1a(T233A) variant is unable to restore basal resistance against $X$. $o$. pv. oryzae in an osptila mutant background in comparison to OsPti1 wildtype (33).

Although much is known about the enzymatic activity and upstream activators of Pti1-like kinases, little is known about their downstream substrates or associated proteins that might regulate their autophosphorylation status. To find potential substrates and regulators of the Ptilb kinase we used a combined FLAG co-immunoprecipitation and Strep pull-down approach coupled with mass spectrometry. We focused our research on Ptilb because previous RNA-Seq analyses indicated that expression of the Ptilb gene and not the Ptila gene is induced by MAMPs csp22 and flgII-28 and infection by P. syringae pv. tomato, suggesting it might play a more prominent role in PTI (34). We found that Pti1b expressed in $N$. benthamiana leaves co-purifies with a phosphatase of the PP2C family (NbPP2C6). We verified this interaction using additional assays and investigated the role of the phosphatase in regulating Ptilb. Our observations indicate that PP2C6 dephosphorylates Ptilb and acts as a negative regulator of PTI signaling.

\section{Experimental procedures}

DNA cloning. The AtPIP2a gene was transferred from plasmid pCambia-AtPIP2a-cLuc (kind gift from G. Coaker) into the pJLSmart Gateway entry vector (40) and then moved into the pGWB-SF destination plasmid that enables protein expression in fusion with a $2 x$ Strep-FLAG- at the $\mathrm{C}$ terminus of the protein. pGWB-SF plasmid was obtained by cloning a 2xStrep sequence into pGWB411 vector (41). Ptilb(K96N)-pJLSmart and Ptilb(R234C)-pJLSmart were generated with PCR-based site-directed mutagenesis with construct Ptilb-pJLSmart (26) as the template. Ptilb, Ptilb(K96N) and Ptilb(R234C) genes were then transferred from pJLSmart into the pGWB-SF destination plasmid. For protein expression in E. coli, the Ptilb gene was amplified from Pti1b-pJLSmart with primers that enabled 
PP2C6 phosphatase and plant immunity

cloning into the pET30a+ vector using restriction enzyme NdeI and NotI. Ptilb(K96N), Ptilb(S232A), Ptilb(T233A), Ptilb(S232A,T233A), Ptilb(T233D), Ptilb(R234C) and Ptilb(T233A,R234C) variants were generated with PCR-based site-directed mutagenesis. The appropriate Ptilb variant in the pET30a+ plasmid was used as the template for the PCR. The PP2C6 gene was amplified from tomato cDNA and cloned into pASK3 plasmid using restriction enzymes $X b a \mathrm{I}$ and $A f e \mathrm{I}$. The $P P 2 C 6(\mathrm{NN})$ variant was generated with PCR-based site-directed mutagenesis with PP2C6-pASK3 as the template. For ROS assays in N. benthamiana PP2C6 variants were amplified from pASK3 plasmids and cloned into pJLTRBO (42) with restriction enzymes NotI and AvrII. Similarly, the GFP gene was amplified from plasmid pGWB505 and cloned into the pJLTRBO plasmid using the same restriction enzymes. To carry out the split luciferase complementation assay (SLCA) Ptilb and PP2C6 genes were amplified with PCR and cloned into pCambia-nLuc and pCambia-cLuc respectively using restriction enzymes KpnI and SalI. All primers used in this study are listed in Supplementary Tables S2 and S3.

Bacteria growth and infiltration. Agrobacterium tumefaciens GV3101 was grown on LB medium with appropriate antibiotics for 48 hours at $30^{\circ} \mathrm{C}$. The bacterial cells were collected and suspended in buffer containing: $10 \mathrm{mM}$ MES, $10 \mathrm{mM} \mathrm{MgCl} 2,200 \mu \mathrm{M}$ acetosyringone, $\mathrm{pH}$ 5.7. For agroinfiltration into $N$. benthamiana leaves, bacteria were diluted to OD 1.0, and incubated for 3 hours at room temperature. Nicotiana benthamiana leaves were infiltrated with a needleless syringe.

Protein expression and purification. For mass spectrometry analysis, proteins in fusion with 2xStrepFLAG were purified with Anti-FLAG M2 Magnetic Beads (Sigma Co.). Purification was carried out according to the manufacturer's protocol with minor modifications. Specifically, $5 \mathrm{~g}$ of $N$. benthamiana leaf tissue was ground in liquid nitrogen and dissolved in $7.5 \mathrm{ml}$ of extraction buffer containing: $150 \mathrm{nM}$ Tris-Cl, $150 \mathrm{mM} \mathrm{NaCl}, 5 \mathrm{mM}$ DTT, $10 \mathrm{mM} \mathrm{MnCl2,} \mathrm{1 \%} \mathrm{[v/v]} \mathrm{Triton} \mathrm{X-100,} \mathrm{PhosSTOP}{ }^{\mathrm{TM}}$ - phosphatase inhibitor tablets (Sigma), cOmplete ${ }^{\mathrm{TM}}$, Mini, EDTA-free Protease Inhibitor Cocktail (Roche Co.), pH 7.4). After $30 \mathrm{~min}$ incubation on a rotator at $4^{\circ} \mathrm{C}$ the protein samples were centrifuged at $18,000 \mathrm{xg}$ for 30 min to remove plant debris and the protein extract was filtered through Miracloth filter paper (Calbiochem Co.). Next, $20 \mu \mathrm{l}$ of previously washed anti-FLAG magnetic beads in an amount equivalent to the $20 \mu 1$ of magnetic bead suspension was added to the protein extract. The sample was incubated on a rotator for 1 hour at $4{ }^{\circ} \mathrm{C}$ and the magnetic beads were then collected using a magnetic rack. The beads were washed 3 times with $200 \mu 1$ extraction buffer and eluted with $100 \mu 1$ of elution buffer containing: $100 \mathrm{mM}$ Tris-Cl, $150 \mathrm{mM} \mathrm{NaCl}, 5 \mathrm{mM}$ DTT, $10 \mathrm{mM} \mathrm{MnCl} 2, \mathrm{pH} 8.0)$ and $450 \mathrm{ng} / \mu 1$ 3xFLAG peptide. After elution, MagStrep "type 3" XT beads (https://www.iba-lifesciences.com) in an amount equivalent to the $10 \mu 1$ of magnetic bead suspension was added to the sample. After 1.5 hours of incubation on a rotator at $4^{\circ} \mathrm{C}$ the beads were washed two times with $200 \mu 1$ of washing buffer containing: $100 \mathrm{mM}$ Tris-Cl, 150 
PP2C6 phosphatase and plant immunity

$\mathrm{mM} \mathrm{NaCl}, 5 \mathrm{mM}$ DTT, $\mathrm{pH}$ 8.0). The proteins bound to the beads were then eluted by boiling in Laemmli buffer.

To purify 6xHis-tagged proteins a frozen pellet of $E$. coli strain DE3(pLys)Rosetta was thawed in buffer containing: $50 \mathrm{mM}$ Tris-Cl, $1 \mathrm{M} \mathrm{NaCl}_{2}, 10 \mathrm{mM}$ Imidazole, $5 \%$ glycerol, cOmplete ${ }^{\mathrm{TM}}$ Mini EDTA-free Protease Inhibitor Cocktail (Roche Co.), $1 \mathrm{mg} / \mathrm{ml}$ lysozyme, pH8.0). After sonication the protein extract was centrifuged at $18,000 \mathrm{x} g$ for $30 \mathrm{~min}$ to remove bacterial debris. The supernatant was incubated on a rotator with Ni-NTA Agarose (Qiagen Co.) for 1.5 hours at $4^{\circ} \mathrm{C}$. Next, the sample was loaded onto a column and after liquid removal the resin was washed three times with $5 \mathrm{ml}$ of buffer containing: $100 \mathrm{mM}$ Tris-Cl, $1 \mathrm{M} \mathrm{NaCl}_{2}, 10 \%$ glycerol, $10 \mathrm{mM}$ imidazole, $\mathrm{pH}$ 8.0). After washing, the protein bound to the resin was eluted with buffer containing: $50 \mathrm{mM}$ Tris-Cl, $1 \mathrm{M} \mathrm{NaCl}_{2}, 10 \%$ glycerol, $250 \mathrm{mM}$ imidazole, $\mathrm{pH}$ 8.0). For purification of Strep-tagged proteins, frozen E. coli strain DE3(pLys)Rosetta cells were thawed in buffer containing: $150 \mathrm{mM}$ Tris-Cl, $150 \mathrm{mM} \mathrm{NaCl}_{2}, 5 \mathrm{mM}$ DTT, cOmplete ${ }^{\mathrm{TM}}$ Mini EDTA-free Protease Inhibitor Cocktail (Roche) pH 8.0). After sonication, the protein extract was centrifuged 18,000xg for 30 min to remove bacterial debris. The supernatant was incubated on a rotator with Strep-Tactin Macroprep resin (IBA Co.) for 1.5 hours at $4^{\circ} \mathrm{C}$. Next, the resin was washed 3 times with $5 \mathrm{ml}$ of buffer: $150 \mathrm{mM}$ Tris-Cl, $150 \mathrm{mM} \mathrm{NaCl} 2,5 \mathrm{mM}$ DTT $\mathrm{pH} 8.0$, next the proteins bound to the resin were eluted with buffer containing: $150 \mathrm{mM}$ Tris-Cl, $150 \mathrm{mM} \mathrm{NaCl}_{2}, 5 \mathrm{mM}$ DTT, $2.5 \mathrm{mM}$ biotin, $\mathrm{pH} 8.0$ ).

Western blotting. Protein samples were separated in $10 \%$ bisacrylamide gels at $200 \mathrm{~V}$. Detection of phosphorylated proteins was performed in blocking solution (5\% BSA, 0.1\% [v/v] Tween-20) with antiphosphothreonine antibodies (9381, Cell Signaling) and with anti-rabbit-HRP (W4011, Promega). Detection of tagged proteins was carried out in blocking solution ( $5 \%$ fat-free milk, $0.5 \%$ [v/v] Tween20). Other antibodies used include: StrepMAB-Classic, HRP conjugate (2-1509-001, IBA), anti-HA High Affinity (11867423001, Roche), anti-polyHistidine antibody (H1029, Sigma) ANTI-FLAG® M2 (F1804, Sigma), anti-first 258 amino acids of luciferase (NB600, Novus), anti-C-terminal part of luciferase (sc74548, Santa Cruz Biotechnology), anti-GFP (11814460001, Roche), anti-rat-HRP (sc-2006, Santa Cruz Biotechnology), and anti-mouse-HRP (sc2005, Santa Cruz Biotechnology).

Mass spectrometry analysis. Peptide mixtures were analyzed by LC-MS-MS/MS (liquid chromatography coupled to tandem mass spectrometry) using Nano-Acquity (Waters) LC system and LTQ-FT-Orbitrap mass spectrometer (Thermo Electron Corp., San Jose, CA). Prior to the analysis, proteins were subjected to a standard "in-solution digestion" procedure during which proteins were reduced with $50 \mathrm{mM}$ TCEP (for $60 \mathrm{~min}$ at $60^{\circ} \mathrm{C}$ ), alkylated with $200 \mathrm{mM}$ MMTS (45 min at room temperature) and digested overnight with trypsin (sequencing grade modified Trypsin - Promega V5111). 
PP2C6 phosphatase and plant immunity

The peptide mixture was applied to RP-18 precolumn (nanoACQUITY Symmetry® C18 - Waters 186003514) using water containing $0.1 \%$ TFA as a mobile phase and then transferred to a nano-HPLC RP-18 column (nanoACQUITY BEH C18 - Waters 186003545) using an acetonitrile gradient (5\% - 35\% $\mathrm{AcN}$ ) for $180 \mathrm{~min}$ in the presence of $0.05 \%$ formic acid with a flowrate of $250 \mathrm{nl} / \mathrm{min}$. Column outlet was directly coupled to the electrospray ion source of the spectrometer working in the regime of data dependent MS to MS/MS switch. A blank run to ensure lack of cross contamination from previous samples preceded each analysis. The data acquired were processed by Mascot Distiller followed by Mascot Search (Matrix Science, London, UK, on-site license) against the database 'NBenthLuteoviridaecRAPBSAProtAmod' is accessible at: $\mathrm{ftp}$ //ftp.solgenomics.net/proteomics/Nicotiana_benthamiana/Cilia_lab/proteomics_db/(Stacy DeBlasio and Michelle Cilia, 2014 personal communication). Search parameters for precursor and product ions mass tolerance were $30 \mathrm{ppm}$ and $0.1 \mathrm{Da}$, respectively, enzyme specificity: trypsin, missed cleavage sites allowed: 1, fixed modification of cysteine by methylthio and variable modification of methionine oxidation. Peptides with Mascot Score exceeding the threshold value corresponding to $<5 \%$ expectation value, calculated by Mascot procedure, were considered to be positively identified. Detected phosphorylated peptides were manually inspected.

Kinase assay. For kinase assays, the proteins were incubated at room temperature in buffer containing: $150 \mathrm{mM}$ HEPES, $150 \mathrm{mM} \mathrm{NaCl} 210 \mathrm{mM} \mathrm{MgCl} 2,10 \mathrm{mM} \mathrm{MnCl} 2,2 \mathrm{mM}$ DTT, $20 \mu \mathrm{M}$ ATP and $1 \mu \mathrm{Ci}$ of $\left[{ }^{32} \mathrm{P}\right] \mathrm{X}$-ATP (Perkin Elmer Co.) $\mathrm{pH}$ 8.0. The total volume of the reaction mixture was $20 \mu 1$. The reaction was terminated by adding Laemmli buffer and boiling for $5 \mathrm{~min}$. The proteins were separated on $10 \%$ polyacrylamide gels and visualized by autoradiography. To confirm protein loading the gels were stained with Coomassie Blue.

Strep-tag pull-down assay. To decrease nonspecific interactions between MagStrep "type 3" XT beads and proteins the beads were incubated in $100 \mu \mathrm{l}$ of buffer containing: $150 \mathrm{mM}$ HEPES, $150 \mathrm{mM} \mathrm{NaCl}_{2} 10$ $\mathrm{mM} \mathrm{MgCl} 2,10 \mathrm{mM} \mathrm{MnCl} 2,2 \mathrm{mM}$ DTT, $20 \mu \mathrm{M}$ ATP, $0.1 \mu \mathrm{g} / \mu 1 \mathrm{BSA}, 1 \%$ Tween, $\mathrm{pH} 8.0$ on a rotator for one hour at $4^{\circ} \mathrm{C}$. Next $30 \mu \mathrm{g}$ of $6 x$ His-tagged Ptilb-variants and PPc6 in fusion with Strep-tag were added to the solution with the beads. Samples were incubated for 2 hours on a rotator at $4{ }^{\circ} \mathrm{C}$ and the beads were collected using a magnetic rack and washed 2 times with $300 \mu 1$ of washing buffer containing: $150 \mathrm{mM}$ HEPES, $150 \mathrm{mM} \mathrm{NaCl} 210 \mathrm{mM} \mathrm{MgCl}$, $10 \mathrm{mM} \mathrm{MnCl}_{2}, 2$ mM DTT, $20 \mu \mathrm{M}$ ATP, $1 \%$ Tween, pH 8.0. Next, the beads were resuspended again in the washing buffer and transferred to a new Eppendorf tube. The beads were collected with a magnetic rack and the proteins were eluted by boiling in Laemmli buffer. The obtained samples were separated on $10 \%$ polyacrylamide gels and transferred to a PVDF membrane. 
PP2C6 phosphatase and plant immunity

The presence of 6xHis-tagged proteins and Strep-tagged proteins was tested with 6xHIS TagAntibody HIS.H8 (Invitrogen Co.) and StrepMAB-Classic, HRP conjugate (IBA Co.) respectively.

Split luciferase complementation assay. $N$. benthamiana leaves were infiltrated with A. tumefaciens GV3101 carrying pCambia plasmids with the genes in fusion with the N-terminal or C-terminal part of luciferase (43). Three days after agroinfiltration discs were removed from the leaves using a cork borer size 2 and placed in $100 \mu 1$ water in a well of a 96-well plate. After a one-hour incubation a solution of 2 $\mathrm{mM}$ luciferin in water was added to each well and the relative light units were determined with a luminometer (Biotek Co.) over a period of $45 \mathrm{~min}$. The expression level of recombinant proteins was determined by Western blotting.

Reactive oxygen species assay. ROS assays were performed as described in (44,45). Leaf discs were removed from $N$. benthamiana leaves and incubated overnight in water, in white, flat-bottom, 96-well plates (Greiner Bio-One Co.). After 16 hours the water was replaced with a water solution containing: 34 $\mu \mathrm{g} / \mathrm{ml}$ luminol (Sigma-Aldrich Co.) and $20 \mu \mathrm{g} / \mathrm{ml}$ horseradish peroxidase (type VI-A, Sigma-Aldrich and the designated concentration of flg22 and $\operatorname{csp} 22$. The relative light units were determined with a luminometer (Biotek Co.) over a period of $45 \mathrm{~min}$.

Plant growth conditions. $N$. benthamiana accession $\mathrm{Nb}-1$ (46) was used in all experiments. Nb-1 plants were grown for 4-6 weeks in a controlled environment chamber with 16 hours light, $65 \%$ relative humidity and a temperature of $24^{\circ} \mathrm{C}$ (light period) and $22^{\circ} \mathrm{C}$ (dark period).

Statistical analysis. The normality of the data was assessed using normal quantile plots (q-q plots) and the Shapiro-Wilk test. The equality of the variance was as assessed using the Bartlett test and variance of the samples is indicated by the standard deviation represented by error bars. Welch's or Tukey-Kramer tests were used to test the differences between the means, $p$ values are indicated in the Figure legends. Statistical analysis was performed with JMP Pro 14 software.

\section{Results}

\section{Pti1b interacts with phosphatase PP2C6 in vitro and in plant cells}

To identify plant proteins that interact with Pti1b, constructs encoding the kinase and an inactive variant Pti1b(K96N), both with a C-terminal 2xStrep-FLAG epitope tag, were individually introduced into leaves of $N$. benthamiana using Agrobacterium-mediated transient transformation (agroinfiltration) and Pti1b proteins were purified with FLAG-immunoprecipitation followed by Strep pull-down (Supplementary 
PP2C6 phosphatase and plant immunity

Fig. S1). Pti1b has S-acylation sites in its $\mathrm{N}$ terminus which play a role in its localization to the cell periphery (26). As a control, therefore, we applied the same purification method using another membraneassociated protein from Arabidopsis, PIP2A (AT3G53420) (35). The samples obtained were submitted for mass spectrometry analysis to identify potential Ptilb-interacting proteins. A protein was considered a candidate interactor if a derived peptide was detected in at least two samples with Ptilb or Pti1b(K96N) and was absent in all PIP2a samples (Fig. 1A).

One of the proteins that met these criteria was a protein phosphatase $2 \mathrm{C}$ with close similarity to tomato phosphatase Solyc07g066260. Phylogenetic analysis indicated that the closest protein in Arabidopsis to tomato phosphatase Solyc07g066260 is Arabidopsis phosphatase AtPP2C6 (At1g16220) (36), and we will refer hereafter to this tomato phosphatase as PP2C6 (Supplementary Fig. S2). Supporting a role for this phosphatase in PTI, the expression of the PP2C6 gene is induced in tomato in response to treatment with MAMPs flgII-28 and csp22 (Supplementary Table S1).

To confirm the interaction between Pti1b and PP2C6, we first used a Strep pull-down assay in combination with wild type Ptilb, its kinase-inactive form and two variants with substitutions at threonine-233, the major autophosphorylation site in the Ptila kinase (28). Each Ptilb protein was purified as a 6xHIS-tagged fusion, and incubated in kinase buffer with PP2C6-Strep. After incubation, PP2C6-Strep was purified with Strep-affinity chromatography and the possible presence of Pti1b-6xHIS was tested with an anti-6xHIS antibody. As shown in Fig. 1B, each of the Pti1b variants was detected only in samples containing PP2C6.

As further confirmation we examined the interaction of Pti1b and PP2C6 in plant cells using a splitluciferase complementation assay. Pti1b-nLuc and PP2C6-cLuc or Pti1b-nLuc and cLuc-AtPIP2a (as a negative control) were transiently expressed in $N$. benthamiana leaves using agroinfiltration and the leaf discs were treated with $1 \mathrm{mM}$ luciferin. We detected statistically significantly more luminescence from the leaf discs expressing Ptib-nLuc and PP2C6-cLuc than from leaf discs expressing the negative control (Fig. 1C). Western blotting confirmed expression of each of the nLuc and cLuc proteins (Fig. 1D). From these experiments, we conclude that Ptilb interacts with protein phosphatase PP2C6 and, at least in vitro, this interaction occurs independently of Ptilb autophosphorylation.

\section{Pti1b autophosphorylates in vitro on threonine-233}

In previous work we showed that the Ptila kinase, a homolog of Pti1b, has protein kinase activity and threonine-233 in its activation loop is its major autophosphorylation site (28). The present study focused on Ptilb because a subsequent RNA-Seq experiment found that expression of the Ptilb gene is induced 
PP2C6 phosphatase and plant immunity

by MAMPs whereas Ptila is not (Supplementary Table S1). We therefore tested Ptilb and its presumed kinase-inactive variant for kinase activity in an in vitro assay. Wild type Ptilb strongly autophosphorylated whereas no phosphorylation was detected with the Pti1b(K69N) variant (Fig. 2A). Next, using LC-MS/MS, we investigated whether threonine-233 is also the major phosphorylation site in Ptilb as it is in Ptila. However, we were unable to detect the ${ }_{230} \mathrm{LHSTR}_{234}$ peptide generated after trypsin or pepsin digestion in either phosphorylated or unphosphorylated form.

To overcome this obstacle, we generated a Pti1b variant where the arginine-234 at the trypsin digestion site was substituted to cysteine. This variant was based on a recent report that the MARIS kinase from Arabidopsis, which belongs to the same subfamily VIII of RLCKs as Ptilb, gains constitutive function when it carries the analogous arginine-to-cysteine substitution (R240C; Fig. 2B) (18). Such a modified Ptilb, when digested with trypsin, would generate a longer peptide containing threonine-233 (LHSTCVLGTFGYHAPEYAMTGQLSSK) that might be more easily detected by LC-MS/MS. Indeed, with LC-MS/MS analysis of the autophosphorylated Pti1(R234C) we observed the presence of both phosphorylated and unphosphorylated forms of the longer peptide. Both threonine-233 and, unexpectedly, serine-232 were found to be phosphorylated in this peptide (Fig. 2C, Supplementary Fig. S3).

To investigate this observation further, we created a series of Ptilb variants with substitutions at serine232 and threonine-233: Pti1b(S232A), Pti1b(T233A), Pti1b(S232A,T233A) and a potential phosphomimic substitution, Ptilb(T233D). These Pti1b variants were expressed in E. coli, purified and incubated in kinase buffer. Ptilb wild type and Ptilb(K96N) were used as a positive and negative control respectively. The results obtained with autoradiography showed that substitution of threonine-233 to either alanine or aspartic acid greatly decreased the autophosphorylation of Pti1b (Fig. 2D). In contrast, the S232A substitution had no impact on autophosphorylation of Ptilb. These data indicate that threonine-233 is likely the major site of autophosphorylation in Pti1b and suggest that the R234C substitution may cause enhanced phosphorylation of S232.

\section{PP2C6 dephosphorylates Ptilb in vitro and in plant cells}

The interaction of PP2C6 with Ptilb suggests the phosphatase might act to dephosphorylate the kinase possibly as a mechanism of desensitizing its activated state. To test this possibility, we incubated Ptilb6xHIS in kinase buffer, either alone or in the presence of PP2C6-Strep or a variant of PP2C6-Strep that has the aspartate residues at positions 110 and 234 substituted to asparagine [PP2C6(NN)-Strep]. Such asparagine substitutions are known to abolish phosphatase activity of PP2C proteins (23). We observed that in the presence of PP2C6-Strep the phosphorylated form of Ptilb was greatly reduced as compared to Pti1b incubated alone or in the presence of the inactive variant PP2C6(NN)-Strep (Fig. 3A). 
PP2C6 phosphatase and plant immunity

We further examined whether PP2C6 affects Pti1b phosphorylation in vivo. Using agroinfiltration we coexpressed Pti1b-2xStrep-FLAG with either PP2C6-3xHA or PP2C6(NN)-3xHA in leaves of $N$.

benthamiana. Six days after agroinfiltration the plant tissue was treated with $1 \mathrm{uM}$ flg22 for 10 minutes.

Ptilb was then purified with Strep-affinity chromatography and the phosphorylation level of Ptilb was tested with antibodies that specifically recognize phosphorylated threonine residues. The specificity of the antibodies for phosphorylated Ptilb was tested with different in vitro phosphorylated Ptilb variants (Supplementary Fig. S4). We observed that the phosphorylation level of Pti1b-2xStrep-FLAG coexpressed with PP2C6-3xHA is reduced in comparison to Pti1b co-expressed with PP2C6(NN)-3xHA (Fig. 3B). Collectively, the data from Figs. 2 and 3 indicate that PP2C6 dephosphorylates threonine-233, the major in vitro autophosphorylation site in Ptilb.

\section{Pti1b(R234C) is recalcitrant to PP2C6 phosphatase activity in vitro}

The arginine-to-cysteine substitution at position 240 in MARIS causes the protein to gain a constitutiveactive function which restores pollen tube cell wall integrity in an anxl/anx2 Arabidopsis mutant (18). The underlying reason for this gain-of-function activity is unknown $(23,37)$. The corresponding arginine in Pti1b, at position 234, is adjacent to threonine-233 and we speculated that a R234C substitution might impact the kinase activity of Ptilb. To test this possibility, we compared the autophosphorylation activity of Ptilb and Pti1b(R234C) in an in vitro kinase assay and found no difference between them (Fig. 4A). We next examined whether the R234 substitution might impact the activity of PP2C6 towards Pti1b. The kinase activity of Pti1b and Pti1b(R234C) were assayed in the presence of PP2C6, PP2C6(NN), calf intestinal phosphatase (CIP) or shrimp alkaline phosphatase (rSAP). Remarkably, PP2C6 dephosphorylated Pt1b but was incapable of dephosphorylating Pti1b(R234C) (Fig. 4B). Both Pti1b proteins were effectively dephosphorylated by CIP and rSAP (Fig. 4B); for unknown reasons, CIP was phosphorylated independently of Ptilb (Supplementary Fig. S5). We next investigated whether the inability of PP2C to dephosphorylate Pti1b(R234C) was due to the loss of an interaction between these two proteins. Using the Strep pull-down assay described above we found that PP2C6-Strep interacted just as well with Pti1b as it did with Pti1b(R234C) (Fig. 4C). Thus, the Pti1b(R234C) variant has enzymatic activity comparable to Pti1b and still interacts with PP2C6 although it resists PP2C6 phosphatase activity.

\section{Peptide flg22 induces the phosphorylation of Pti1b on threonine residue(s) in plant cells}

Perception of MAMPs like peptide flg22 by PRRs induces phosphorylation of downstream signaling components $(13,15)$. To test possible MAMP induction of Ptilb phosphorylation we chose to use Pti1b(R234)-Strep to avoid dephosphorylation by PP2C6. Pti1b(R234)-Strep was expressed in N. benthamiana leaves using agroinfiltration and the leaf tissue was infiltrated 3 days later with $1 \mu \mathrm{M}$ flg22 
PP2C6 phosphatase and plant immunity

or with water as a control. Ten minutes after addition of flg22 the tissue was collected and Pti1b(R234C)

protein was purified by Strep-affinity chromatography. One half of the purified protein was then incubated with CIP to remove phosphate from Pti1b(R234C) and the samples were analyzed by Western blotting using the antibody that specifically detects phosphorylated threonine residues. This analysis revealed a higher level of phosphothreonine on Pti1b(R234C) from flg22-treated leaf tissue than from the control treated with water alone (Fig. 5). In turn, Pti1b(R234C) protein purified from the water-treated leaf tissue was phosphorylated to a higher level than the CIP-treated samples indicating that Ptilb is phosphorylated at a basal level even without flg22 treatment.

\section{PP2C6 decreases the production of reactive oxygen species generated in leaves in response to flg22 and $\operatorname{csp} 22$}

Tomato plants with decreased levels of Ptila and Ptilb produce less ROS after treatment with flg22 compared to wild type plants (26). We hypothesized that the dephosphorylation of Pti1b by PP2C6 might negatively regulate the role of Ptil in generating ROS in response to flg22. To test this hypothesis, we used agroinfiltration to overexpress PP2C6-3xHA in leaves of $N$. benthamiana, which has four Pti1 homologs (26), and measured the production of ROS in response to flg22 and another MAMP, csp22. As controls, we overexpressed the inactive PP2C6(NN)-3xHA variant. Leaf discs expressing PP2C6 produced statistically significantly lower levels of ROS after treatment with flg 22 and $\operatorname{csp} 22$ compared to leaf disks expressing PP2C6(NN)-3xHA (Fig. 6AC). Each of the proteins was shown to be expressed well by Western blotting (Fig. 6BD). PP2C6 therefore acts as a negative regulator of ROS production in plant leaves, likely by dephosphorylating Ptilb.

\section{Discussion}

We reported previously that the Pti1 kinases are activators of ROS burst in tomato and positive regulators of disease resistance against $P$. syringae pv. tomato (26). Here we identified PP2C6 as an interactor of Pti1 with the ability to dephosphorylate the major Ptilb autophosphorylation site, threonine-233. Phosphorylation of Pti1b is induced upon treatment of leaves with MAMPs and overexpression of PP2C6 in leaves greatly reduces production of immunity-associated ROS. Below we elaborate on our key findings about Pti1b phosphorylation status, the role of PP2C6 in regulating Pti1b, and on the ability of the arginine-234-cysteine substitution to make Ptilb recalcitrant to PP2C6 activity.

\section{Pti1b phosphorylation}

Our mass spectrometry experiments suggested that Ptilb is autophosphorylated in vitro on threonine-233 and/or on serine-232. However, with autoradiography we were only able to find evidence for Pti1b 
PP2C6 phosphatase and plant immunity

phosphorylation on threonine-233 and our current data therefore supports this residue as the major phosphorylation site. Phosphorylation of serine-232 was also not observed in a previous study that reported threonine-233 as the major site of phosphorylation in the Pti1a kinase (28). Interestingly, we do observe weak phosphorylation associated with both Pti1b(T233A) and Pti1b(T233D). The source of this signal could be phosphorylated serine-232 which may be a minor site of autophosphorylation in Pti1b. It is possible that the arginine-234-cysteine substitution causes more phosphorylation to occur on serine-232 than occurs in wildtype Pti1b. This possibility is supported by the greater number of peptides observed by mass spectrometry with phosphorylated S232 (Fig. 2C) and by the experiment in which we compared the autophosphorylation of Pti1b(T233A) and Pti1b(T233A/R234C) and found the latter protein to be more strongly phosphorylated (Supplementary Fig. S4A). It also appears that the serine-232-alanine substitution might strengthen the autophosphorylation on threonine-233 as shown in Fig. 2B.

We found that flg22 induces the phosphorylation of Pti1b on threonine residue(s) in N. benthamiana. Although we have not investigated this further yet, it is likely this phosphorylation occurs on threonine233 because that residue is the major phosphorylation site in vitro. It has been reported previously that OsPtila is phosphorylated in rice cells on its threonine-233 (corresponding to threonine-233 in Pti1b) (38). Additionally, it was shown that threonine-233 of OsPtila plays a role in immunity against $X . o$. pv. oryzae (39). Further insights into which residues of Ptilb are phosphorylated in plant cells will come from the future development and biochemical characterization of transgenic tomato plants with phosphonull and phosphomimic variants of threonine-233.

\section{The role of phosphatase PP2C6}

There are relatively few protein phosphatases which are known regulate RLCKs that are activators of plant immune responses. One of these, PP2C38 (23), which regulates the immunity-associated RLCK BIK1, has some similarities to PP2C6. Like PP2C6 and Pti1b, PP2C38 negatively controls the phosphorylation status of BIK1 (23). Treatment of Arabidopsis leaves with flg22 induces BIK1 activity causing it to phosphorylate PP2C38 which leads to dissociation of the phosphatase from BIK1 (23). The dissociated BIK1 is then able to activate downstream targets. We did not observe Ptilb phosphorylation of either PP2C6 or PP2C6(NN) in vitro. Therefore, we have no evidence that release of Ptilb from interaction with PP2C6 relieves negative regulation by PP2C6 indicating Pti1b-PP2C6 may use a different mechanism than BIK1-PP2C38. A hypothesis that would be interesting to explore is that Ptilb might be phosphorylated by an upstream kinase such as Oxi1 which allows Pti1b to mitigate the negative regulation imposed by PP2C6. 
PP2C6 phosphatase and plant immunity

\section{The influence of the arginine-234-cysteine substitution on PP2C6 activity}

The MARIS protein, which is a member of the same RLCK subfamily as Ptilb, acts as a regulator of cell wall integrity in Arabidopsis (18). The variant of this kinase with an arginine-to-cysteine substitution was identified as a constitutive-active version of MARIS but the underlying reason for this activity is unknown (18). Here we found that the analogous substitution in Ptilb (R234C) makes the kinase insensitive to dephosphorylation by PP2C6 although its kinase activity is comparable to Ptilb wild type. Based on this observation, it is possible that MARIS(R240C) is also able to resist dephosphorylation by an associated phosphatase that normally negatively regulates MARIS. This could explain how the constitutively-active variant of MARIS is able to restore pollen tube cell wall integrity in the absence of Anx1/Anx2.

\section{A model for Ptilb and PP2C6}

Based on the results presented here we propose a model for Ptilb and PP2C6 in pattern-triggered immunity (Supplementary Fig. S6). Various MAMPs including flg22 and csp22 activate PRRs by binding to their extracellular leucine-rich repeat domains. These PRRs act in concert with the co-receptor BAK1 (SERK3B) in signaling pathway(s) which lead to multiple defense responses including production of ROS. Ptilb is proposed to act downstream or with PRR signaling complexes and plays a role in ROS production. Phosphorylation of threonine-233 in Ptilb is proposed to contribute to this mechanism and PP2C6 acts to dephosphorylate this residue thereby inactivating Pti1b and desensitizing the associated signaling pathway. In the future we will investigate the possibility that Ptilb is present in PRR complexes, how it might be impacted by activated PRRs, and search for its potential substrates which are expected to include proteins involved in ROS production.

\section{Acknowledgments}

We thank Fabio Rinaldi and Robyn Roberts helpful comments on the manuscript, Konrad Thorner, Sam Wolfe, and Lydia Zamidar for technical assistance, and Gitta Coaker for the cLuc-PIP2A plasmid. This research was supported by National Science Foundation grant nos. IOS-1451754 and IOS-1546625 (GBM). 
PP2C6 phosphatase and plant immunity

\section{Declaration of interest}

The authors declare that they have no conflicts of interest with the contents of this article.

\section{Author contributions}

FG and GBM conceived and designed the experiments, FG performed experiments, FG and GBM analyzed the data and wrote the paper 


\section{References}

1. Chinchilla, D., Zipfel, C., Robatzek, S., Kemmerling, B., Nurnberger, T., Jones, J. D., Felix, G., and Boller, T. (2007) A flagellin-induced complex of the receptor FLS2 and BAK1 initiates plant defence. Nature 448, 497-500

2. Couto, D., and Zipfel, C. (2016) Regulation of pattern recognition receptor signalling in plants. Nat Rev Immunol 16, 537-552

3. Boller, T., and Felix, G. (2009) A renaissance of elicitors: perception of microbe-associated molecular patterns and danger signals by pattern-recognition receptors. Annu Rev Plant Biol 60, $379-406$

4. Gomez-Gomez, L., and Boller, T. (2000) FLS2: an LRR receptor-like kinase involved in the perception of the bacterial elicitor flagellin in Arabidopsis. Mol Cell 5, 1003-1011

5. Zipfel, C., Robatzek, S., Navarro, L., Oakeley, E. J., Jones, J. D., Felix, G., and Boller, T. (2004) Bacterial disease resistance in Arabidopsis through flagellin perception. Nature 428, 764-767

6. Hind, S. R., Strickler, S. R., Boyle, P. C., Dunham, D. M., Bao, Z., O'Doherty, I. M., Baccile, J. A., Hoki, J. S., Viox, E. G., Clarke, C. R., Vinatzer, B. A., Schroeder, F. C., and Martin, G. B. (2016) Tomato receptor FLAGELLIN-SENSING 3 binds flgII-28 and activates the plant immune system. Nat Plants 2, 16128

7. Wang, L., Albert, M., Einig, E., Furst, U., Krust, D., and Felix, G. (2016) The pattern-recognition receptor CORE of Solanaceae detects bacterial cold-shock protein. Nat Plants 2, 16185

8. Schulze, B., Mentzel, T., Jehle, A., Mueller, K., Beeler, S., Boller, T., Felix, G., and Chinchilla, D. (2010) Rapid heteromerization and phosphorylation of ligand-activated plant transmembrane receptors and their associated kinase BAK1. J Biol Chem 285, 9444-9451

9. Zipfel, C., and Oldroyd, G. E. (2017) Plant signalling in symbiosis and immunity. Nature 543, 328-336

10. Birkenbihl, R. P., Kracher, B., Ross, A., Kramer, K., Finkemeier, I., and Somssich, I. E. (2018) Principles and characteristics of the Arabidopsis WRKY regulatory network during early MAMPtriggered immunity. Plant J 96, 487-502

11. Kadota, Y., Sklenar, J., Derbyshire, P., Stransfeld, L., Asai, S., Ntoukakis, V., Jones, J. D., Shirasu, K., Menke, F., Jones, A., and Zipfel, C. (2014) Direct regulation of the NADPH oxidase RBOHD by the PRR-associated kinase BIK1 during plant immunity. Mol Cell 54, 43-55

12. Underwood, W. (2012) The plant cell wall: a dynamic barrier against pathogen invasion. Front Plant Sci 3, 85

13. Liang, X., and Zhou, J. M. (2018) Receptor-like cytoplasmic kinases: Central players in plant receptor kinase-mediated signaling. Annu Rev Plant Biol 69, 267-299

14. Lu, D., Wu, S., Gao, X., Zhang, Y., Shan, L., and He, P. (2010) A receptor-like cytoplasmic kinase, BIK1, associates with a flagellin receptor complex to initiate plant innate immunity. Proc Natl Acad Sci USA 107, 496-501

15. Li, L., Li, M., Yu, L., Zhou, Z., Liang, X., Liu, Z., Cai, G., Gao, L., Zhang, X., Wang, Y., Chen, S., and Zhou, J. M. (2014) The FLS2-associated kinase BIK1 directly phosphorylates the NADPH oxidase RBOHD to control plant immunity. Cell Host \& Microbe 15, 329-338

16. Yamada, K., Yamaguchi, K., Shirakawa, T., Nakagami, H., Mine, A., Ishikawa, K., Fujiwara, M., Narusaka, M., Narusaka, Y., Ichimura, K., Kobayashi, Y., Matsui, H., Nomura, Y., Nomoto, M., 
bioRxiv preprint doi: https://doi.org/10.1101/613612; this version posted April 18,2019. The copyright holder for this preprint (which was not certified by peer review) is the author/funder, who has granted bioRxiv a license to display the preprint in perpetuity. It is made available under aCC-BY-ND 4.0 International license.

PP2C6 phosphatase and plant immunity

Tada, Y., Fukao, Y., Fukamizo, T., Tsuda, K., Shirasu, K., Shibuya, N., and Kawasaki, T. (2016)

The Arabidopsis CERK1-associated kinase PBL27 connects chitin perception to MAPK activation. EMBO J 35, 2468-2483

17. Lin, Z. D., Liebrand, T. W., Yadeta, K. A., and Coaker, G. L. (2015) PBL13 is a serine/threonine protein kinase that negatively regulates Arabidopsis immune responses. Plant Physiol 169, 29502962

18. Boisson-Dernier, A., Franck, C. M., Lituiev, D. S., and Grossniklaus, U. (2015) Receptor-like cytoplasmic kinase MARIS functions downstream of CrRLK1L-dependent signaling during tip growth. Proc Natl Acad Sci USA 112, 12211-12216

19. Gomez-Gomez, L., Bauer, Z., and Boller, T. (2001) Both the extracellular leucine-rich repeat domain and the kinase activity of FSL2 are required for flagellin binding and signaling in Arabidopsis. Plant Cell 13, 1155-1163

20. Park, C. J., Peng, Y., Chen, X., Dardick, C., Ruan, D., Bart, R., Canlas, P. E., and Ronald, P. C. (2008) Rice XB15, a protein phosphatase 2C, negatively regulates cell death and XA21-mediated innate immunity. PLoS Biol 6, e231

21. He, X., Anderson, J. C., Pozo Od, O., Gu, Y. Q., Tang, X., and Martin, G. B. (2004) Silencing of subfamily I of protein phosphatase 2 A catalytic subunits results in activation of plant defense responses and localized cell death. Plant J 38, 563-577

22. Segonzac, C., Macho, A. P., Sanmartin, M., Ntoukakis, V., Sanchez-Serrano, J. J., and Zipfel, C. (2014) Negative control of BAK1 by protein phosphatase 2A during plant innate immunity. EMBO J 33, 2069-2079

23. Couto, D., Niebergall, R., Liang, X., Bucherl, C. A., Sklenar, J., Macho, A. P., Ntoukakis, V., Derbyshire, P., Altenbach, D., Maclean, D., Robatzek, S., Uhrig, J., Menke, F., Zhou, J. M., and Zipfel, C. (2016) The Arabidopsis protein phosphatase PP2C38 negatively regulates the central immune kinase BIK1. PLoS Pathog 12, e1005811

24. Anderson, J. C., Bartels, S., Gonzalez Besteiro, M. A., Shahollari, B., Ulm, R., and Peck, S. C. (2011) Arabidopsis MAP Kinase Phosphatase 1 (AtMKP1) negatively regulates MPK6-mediated PAMP responses and resistance against bacteria. Plant $J$ 67, 258-268

25. Shubchynskyy, V., Boniecka, J., Schweighofer, A., Simulis, J., Kvederaviciute, K., Stumpe, M., Mauch, F., Balazadeh, S., Mueller-Roeber, B., Boutrot, F., Zipfel, C., and Meskiene, I. (2017) Protein phosphatase AP2C1 negatively regulates basal resistance and defense responses to Pseudomonas syringae. J Exp Bot 68, 1169-1183

26. Schwizer, S., Kraus, C. M., Dunham, D. M., Zheng, Y., Fernandez-Pozo, N., Pombo, M. A., Fei, Z., Chakravarthy, S., and Martin, G. B. (2017) The tomato kinase Ptil contributes to production of reactive oxygen species in response to two flagellin-derived peptides and promotes resistance to Pseudomonas syringae infection. Mol Plant Microbe Interact 30, 725-738

27. Sessa, G., D'Ascenzo, M., Loh, Y.-T., and Martin, G. B. (1998) Biochemical properties of two protein kinases involved in disease resistance signaling in tomato. J Biol Chem 273, 15860-15865

28. Sessa, G., D'Ascenzo, M., and Martin, G. B. (2000) The major site of the Pti1 kinase phosphorylated by the Pto kinase is located in the activation domain and is required for Pto-Pti1 physical interaction. Eur J Biochem 267, 171-178

29. Anthony, R. G., Khan, S., Costa, J., Pais, M. S., and Bogre, L. (2006) The Arabidopsis protein kinase PTI1-2 is activated by convergent phosphatidic acid and oxidative stress signaling pathways downstream of PDK1 and OXI1. J Biol Chem 281, 37536-37546 
PP2C6 phosphatase and plant immunity

30. Forzani, C., Carreri, A., de la Fuente van Bentem, S., Lecourieux, D., Lecourieux, F., and Hirt, H. (2011) The Arabidopsis protein kinase Pto-interacting 1-4 is a common target of the oxidative signal-inducible 1 and mitogen-activated protein kinases. FEBS J 278, 1126-1136

31. Oh, S.-K., Jang, H. A., Lee, S. S., Cho, H. S., Lee, D.-H., Choi, D., and Kwon, S.-Y. (2014) Cucumber Pti1-L is a cytoplasmic protein kinase involved in defense responses and salt tolerance. J Plant Physiol 171, 817-822

32. Takahashi, A., Agrawal, G. K., Yamazaki, M., Onosato, K., Miyao, A., Kawasaki, T., Shimamoto, K., and Hirochika, H. (2007) Rice Ptila negatively regulates RAR1-dependent defense responses. Plant Cell 19, 2940-2951

33. Matsui, H., Yamazaki, M., Kishi-Kaboshi, M., Takahashi, A., and Hirochika, H. (2010) AGC kinase OsOxi1 positively regulates basal resistance through suppression of OsPtila-mediated negative regulation. Plant \& Cell Physiol 51, 1731-1744

34. Rosli, H. G., Zheng, Y., Pombo, M. A., Zhong, S., Bombarely, A., Fei, Z., Collmer, A., and Martin, G. B. (2013) Transcriptomics-based screen for genes induced by flagellin and repressed by pathogen effectors identifies a cell wall-associated kinase involved in plant immunity. Genome Biol 14, R139

35. Qing, D., Yang, Z., Li, M., Wong, W. S., Guo, G., Liu, S., Guo, H., and Li, N. (2016) Quantitative and functional phosphoproteomic analysis reveals that ethylene regulates water transport via the C-terminal phosphorylation of aquaporin PIP2;1 in Arabidopsis. Mol Plant 9 , $158-174$

36. Xue, T., Wang, D., Zhang, S., Ehlting, J., Ni, F., Jakab, S., Zheng, C., and Zhong, Y. (2008) Genome-wide and expression analysis of protein phosphatase 2C in rice and Arabidopsis. BMC Genomics 9, 550

37. Franck, C. M., Westermann, J., Burssner, S., Lentz, R., Lituiev, D. S., and Boisson-Dernier, A. (2018) The protein phosphatases ATUNIS1 and ATUNIS2 regulate cell wall Integrity in tipgrowing cells. Plant Cell 30, 1906-1923

38. Matsui, H., Takahashi, A., and Hirochika, H. (2015) Rice immune regulator, OsPti1a, is specifically phosphorylated at the plasma membrane. Plant Signal Behav 10, e991569

39. Matsui, H., Yamazaki, M., Kishi-Kaboshi, M., Takahashi, A., and Hirochika, H. (2010) AGC kinase OsOxi1 positively regulates basal resistance through suppression of OsPtila-mediated negative regulation. Plant \& cell physiology 51, 1731-1744

40. Mathieu, J., Warthmann, N., Kuttner, F., and Schmid, M. (2007) Export of FT protein from phloem companion cells is sufficient for floral induction in Arabidopsis. Curr Biol 17, 1055-1060

41. Golisz, A., Sikorski, P. J., Kruszka, K., and Kufel, J. (2013) Arabidopsis thaliana LSM proteins function in mRNA splicing and degradation. Nucleic Acids Res 41, 6232-6249

42. Lindbo, J. A. (2007) TRBO: a high-efficiency tobacco mosaic virus RNA-based overexpression vector. Plant Physiol 145, 1232-1240

43. Chen, H., Zou, Y., Shang, Y., Lin, H., Wang, Y., Cai, R., Tang, X., and Zhou, J. M. (2008) Firefly luciferase complementation imaging assay for protein-protein interactions in plants. Plant Physiol 146, 368-376

44. Chakravarthy, S., Velasquez, A. C., Ekengren, S. K., Collmer, A., and Martin, G. B. (2010) Identification of Nicotiana benthamiana genes involved in pathogen-associated molecular pattern-triggered immunity. Mol Plant-Microbe Interact 23, 715-726 
PP2C6 phosphatase and plant immunity

45. Clarke, C. R., Chinchilla, D., Hind, S. R., Taguchi, F., Miki, R., Ichinose, Y., Martin, G. B., Leman, S., Felix, G., and Vinatzer, B. A. (2013) Allelic variation in two distinct Pseudomonas syringae flagellin epitopes modulates the strength of plant immune responses but not bacterial motility. New Phytol 200, 847-860

46. Bombarely, A., Rosli, H. G., Vrebalov, J., Moffett, P., Mueller, L. A., and Martin, G. B. (2012) A draft genome sequence of Nicotiana benthamiana to enhance molecular plant-microbe biology research. Mol Plant-Microbe Interact 25, 1523-1530 
bioRxiv preprint doi: https://doi.org/10.1101/613612; this version posted April 18, 2019. The copyright holder for this preprint (which was not certified by peer review) is the author/funder, who has granted bioRxiv a license to display the preprint in perpetuity. It is made available under

A

\begin{tabular}{|c|c|}
\hline Sample: & $\begin{array}{c}\text { Identified peptides of NbPP2C } \\
\text { phosphatase: }\end{array}$ \\
\hline Pti1b & 0 \\
\hline Pti1b & R.DTLPLLLR.S \\
\hline Pti1b & 0 \\
\hline Pti1b(K96N) & R.DTLPLLLR.S \\
\hline Pti1b(K96N) & 0 \\
\hline Pti1b(K96N) & 0 \\
\hline PIP2A (control) & 0 \\
\hline PIP2A (control) & 0 \\
\hline PIP2A (control) & 0 \\
\hline
\end{tabular}

B

$\begin{array}{rcccccccc}\text { PP2C6-Strep } & + & - & + & - & + & - & + & - \\ \text { Pti1b-6xHIS } & + & + & - & - & - & - & - & - \\ \text { Pti1b(K96N)-6xHIS } & - & - & + & + & - & - & - & - \\ \text { Pti1b(T233A)-6xHIS } & - & - & - & - & + & + & - & - \\ \text { Pti1b(T233D)-6xHIS } & - & - & - & - & - & - & + & +\end{array}$

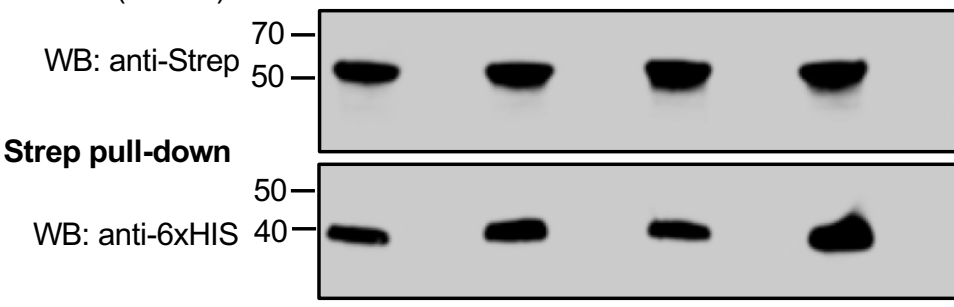

WB: anti-Strep

Input

WB: anti-6xHIS
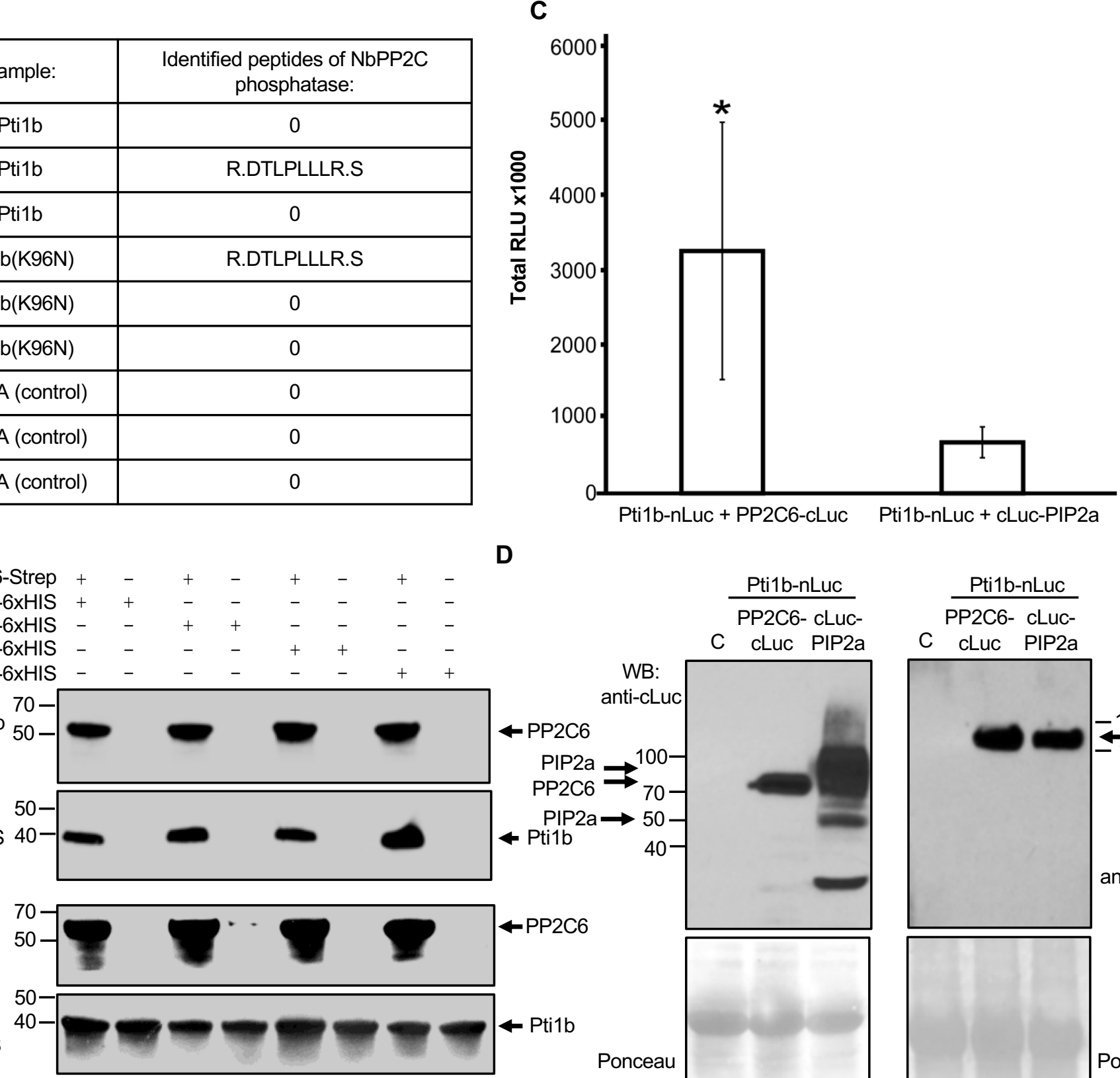

D
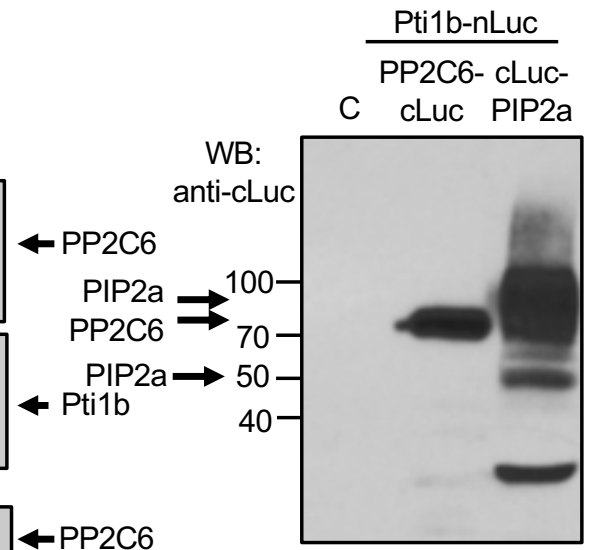

Ponceau
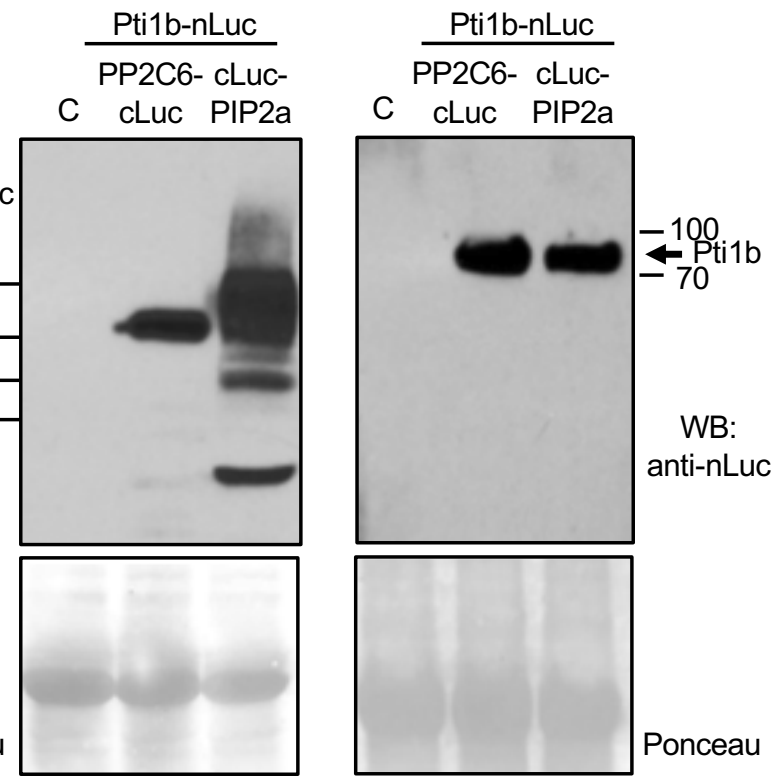

Figure 1. Pti1b interacts with protein phosphatase PP2C6 from tomato in vitro and in plant cells. (A) Pti1b-2xStrep-FLAG was expressed in $N$. benthamiana leaves and purified by affinity chromatography using an anti-FLAG antibody and Strep binding resin to identify Pti1b-interacting proteins. Transmembrane protein PIP2a-2xStrep-FLAG from $A$. thaliana was used as a negative control. The purified proteins were subjected to a tryptic digest and analyzed by MS/MS. Analysis of the spectra with Mascot revealed that Pti1b copurified with a protein phosphatase homologous to Arabidopsis PP2C6 phosphatase (At1g16220) and tomato PP2C6 phosphatase (Solyc07g066260) which we refer to as NbPP2C6. The table shows peptides of NbPP2C6 detected during the mass spectrometry analysis. (B) Variants of Pti1b interact with PP2C6 in vitro. Pti1b-6XHIS variants were expressed and purified from E. coli and then incubated with PP2C6-Strep. After incubation, PP2C6-Strep was purified using Strep affinity chromatography. Pti1b variants were detected by Western blotting (WB) using anti-6xHIS antibody and the PP2C6-Strep was detected with an anti-Strep antibody. The experiment was repeated two times with similar results. (C) Pti1b-nLuc interacts with PP2C6-cLuc but not with cLuc-PIP2a in a split luciferase complementation assay. Graph shows total number of relative light units (RLU) detected over 45 min after treatment of leaf discs with $1 \mathrm{mM}$ luciferin. Bars represent means \pm S.D. ( $n=6)$. Asterisk indicates a significant difference based on Welch's test ( $p$ value $=0.0223$ ). Experiment was repeated three times with similar results. (D) Split-luciferase fusion proteins were detected by Western blotting (WB) using an anti-LUC antibodies after transient expression in N. benthamiana. PIP2a monomer (lower band) and PIP2a dimer (upper band); C, sample prepared from noninfiltrated plant tissue (negative control). To confirm equal loading, the membrane was stained with Ponceau (bottom panels; the prominent protein is Rubisco). 
bioRxiv preprint doi: https://doi org/10.1101/613612; this version posted April 18,2019 . The copyright holder for this preprint (which was not certified by peer review) is the author/funder, who has granted bioRxiv a license to display the preprint in perpetuity. It is made available under aCC-BY-ND 4.0 International license.

A
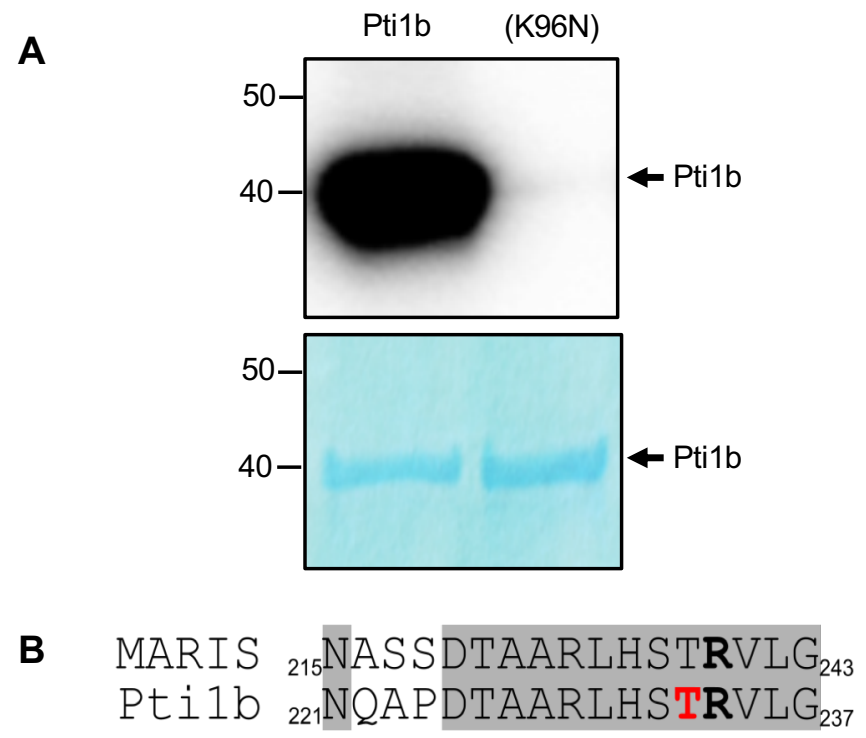

C
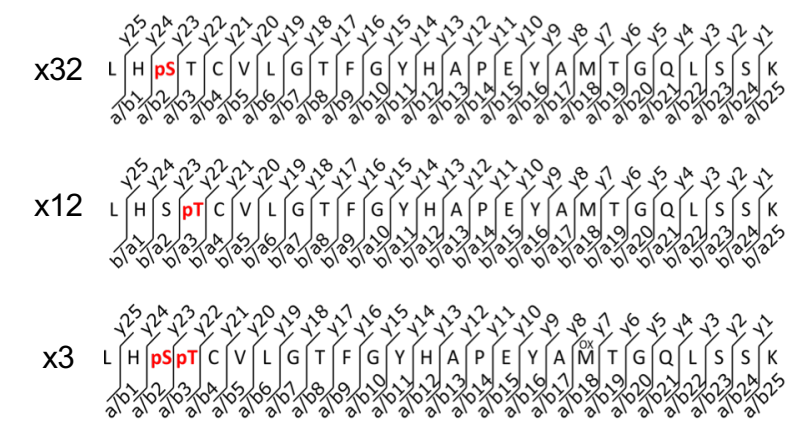

Pti1b
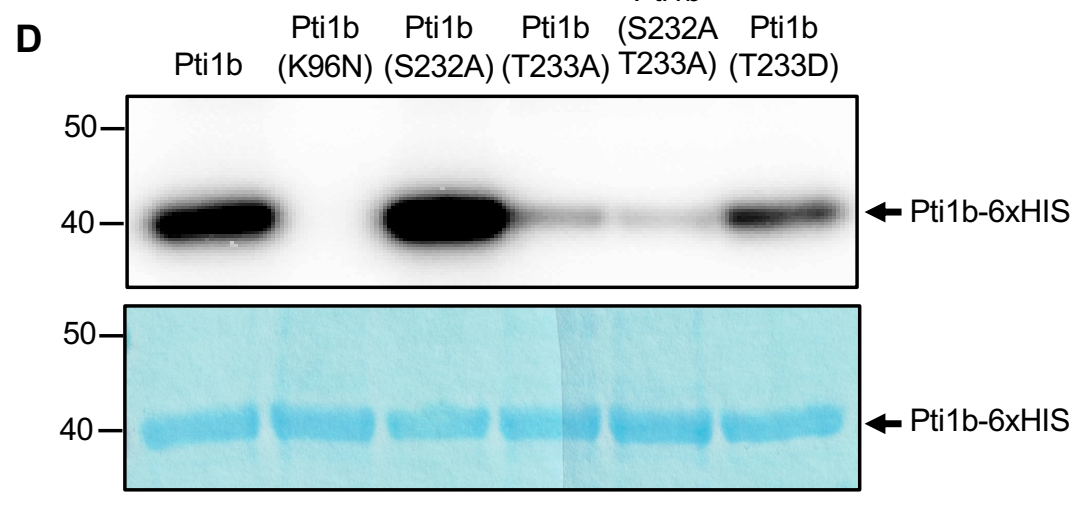

Figure 2. Threonine-233 is the major autophosphorylation site in Pti1b in vitro. (A) Pti1b is an active kinase with autophosphorylation capability. Pti1b and inactive variant Pti1b(K96N) were expressed and purified from E. coli. The proteins were incubated in kinase buffer with ATP- $[\gamma-32]$ and subjected to autoradiography. The experiment was repeated three times with similar results. (B) Alignment of amino acid sequences of Arabidopsis MARIS and Pti1b showing the arginine-240 (bold) in MARIS and corresponding arginine-234 (bold) in Pti1b, and threonine-233, the major site of phosphorylation in Pti1b, in red; identical residues are shown in grey background. (C) Mass spectrometry analysis showed that variant Pti1b(R234C) is phosphorylated on serine-232 and threonine-233. Pti1b(R234C) was phosphorylated in vitro and the phosphorylation sites were mapped with mass spectrometry. The figure shows phosphopeptides detected during the analysis, with phosphorylated residues in red. The numbers on the left indicate the total number of times each phosphopeptide was identified in two independent experiments. Representative spectra for each phosphopeptide are shown in Supplementary Figure S3. (D) Threonine-233 is the major autophosphorylation site in Pti1b. Different variants of Pti1b were produced in $E$. coli and purified with affinity chromatography, and the proteins were incubated in kinase buffer with ATP-[ $\gamma-32]$. The signal from phosphorylated Pti1b was detected with autoradiography and equal loading of the proteins was confirmed with Coomassie blue staining. The experiment was repeated three times with similar results. 
A

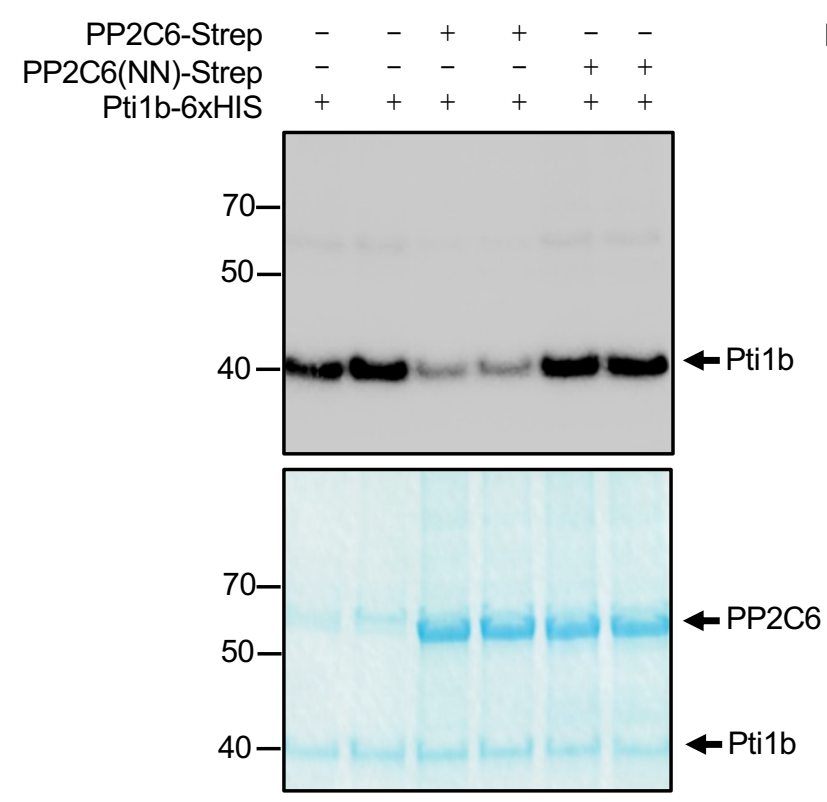

B

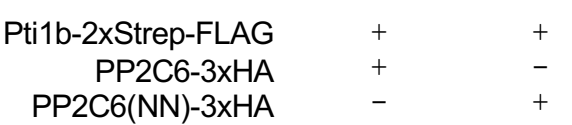

PP2C6(NN)-3XHA $\quad-\quad+$

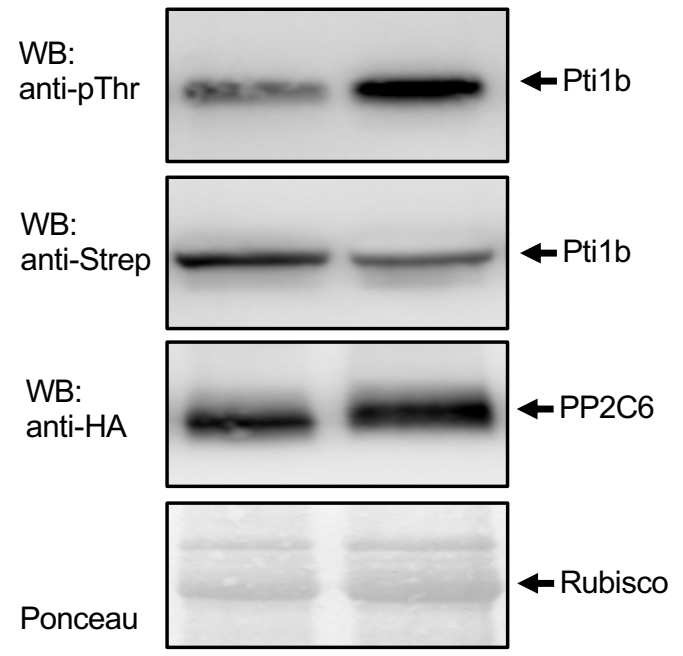

Figure 3. PP2C6 reduces the amount of autophosphorylated Pti1b in comparison to the catalyticallyinactive variant PP2C6(NN) in vitro and in plant cells. (A) PP2C6 dephosphorylates Pti1b in vitro. Pti1b and PP2C6 proteins were produced in E. coli and purified with affinity chromatography. Pti1b was incubated alone or with the two PP2C6 variants in kinase buffer with ATP-[ $\gamma-32]$. The reaction was terminated by boiling the samples in Laemmli buffer. Proteins were separated using gel electrophoresis and phosphorylated Pti1b was detected with autoradiography. Protein amounts were detected with Coomassie blue staining. Two technical repeats for each condition are shown. The experiment was repeated three times with similar results. (B) PP2C6 can reduce phosphorylation status of Pti1b in plant cells. Agroinfiltration was used to co-express Pti1b in leaves of $N$. benthamiana with PP2C6 or PP2C6(NN). Six days after infiltration flg22 was applied and 10 minutes later leaf tissue was harvested and Pti1b was purified using Strep-affinity chromatography. The phosphorylation level of the kinase was detected by Western blotting (WB) with anti-phosphothreonine antibodies. The total amount of Ptilb was determined with anti-Strep antibodies. The presence of PP2C6 variant in crude protein extract used for Pti1b purification was determined with anti-HA antibodies. Rubisco stained with Ponceau was used as a control of protein loading for samples with crude protein extract. 
bioRxiv preprint doi: https://doi.org/10.1101/613612; this version posted April 18,2019 . The copyright holder for this preprint (which was not certified by peer review) is the author/funder, who has granted bioRxiv a license to display the preprint in perpetuity. It is made available under aCC-BY-ND 4.0 International license.

A

Pti1b-6xHIS + -
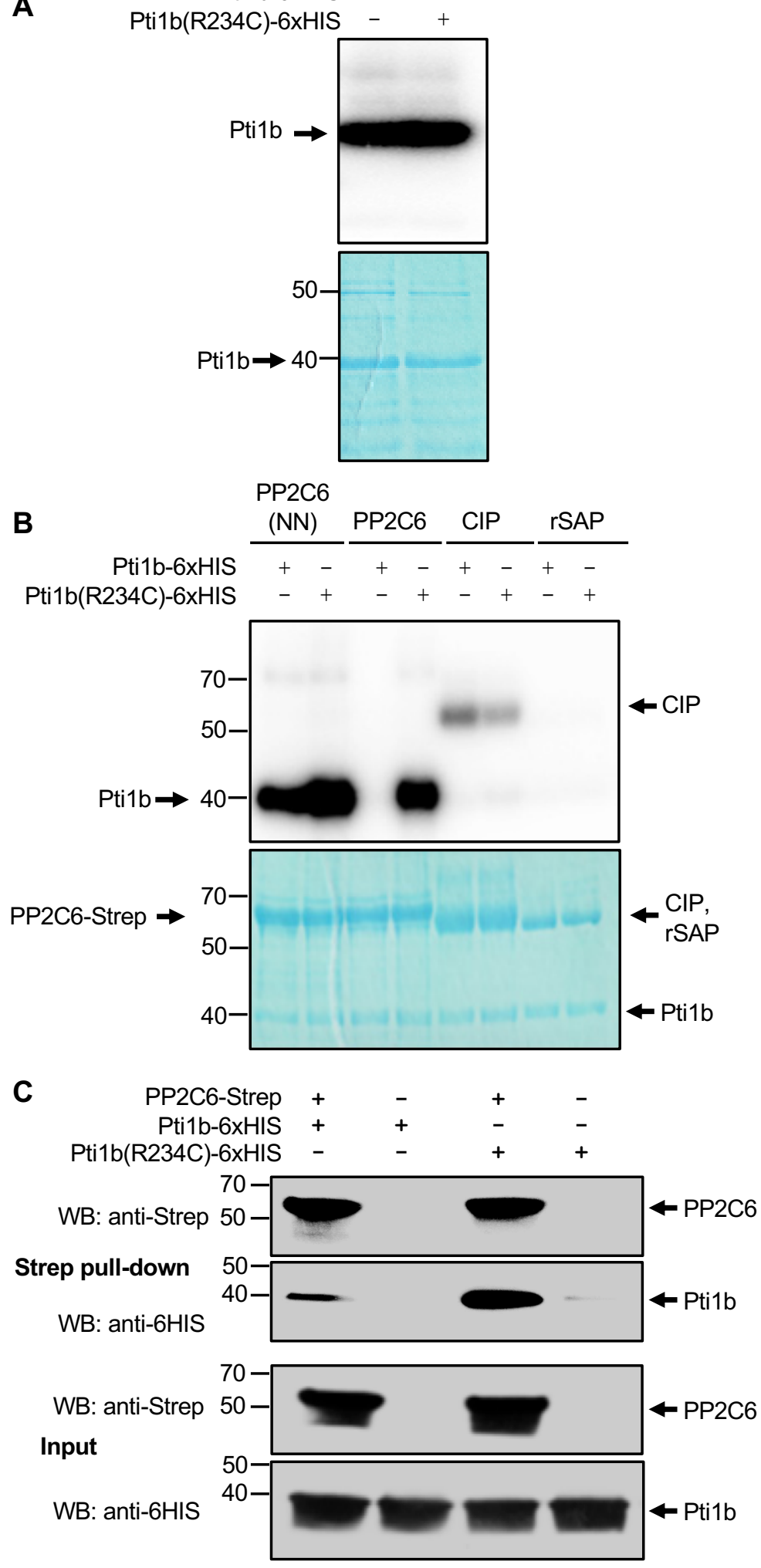

Figure 4. Pti1b(R234C) is recalcitrant to PP2C6 but is sensitive to other phosphatases in vitro. (A) Autophosphorylation levels of Pti1b and Pti1b(R234C) are comparable. Experiment was carried out as in the Figure 2A and experiment was repeated three times with similar results. Lower panel is Coomassie blue-stained gel. (B) Pti1b(R234C) is insensitive to PP2C6. Pti1b and Pti1b(R2324C) were incubated in kinase buffer in the presence of PP2C6(NN), PP2C6, CIP (calf intestinal phosphatase), or rSAP (shrimp alkaline phosphatase) and phosphorylation was detected by autoradiography. The observed phosphorylation of CIP phosphatase is not dependent on Pti1b kinase activity (Supplementary Figure S5). This experiment was repeated three times with similar results. (C) Pti1b(R234C) interacts with PP2C6 in vitro. Proteins were expressed and purified from E. coli. Pti1b variants were then incubated with PP2C6 phosphatase. After incubation PP2C6 phosphatase was purified with Strep-tag affinity chromatography. Pti1b variants were detected by Western blotting (WB) using an anti-6xHIS antibody and PP2C6 was detected with anti-Strep antibody. The experiment was repeated two times with similar results. 


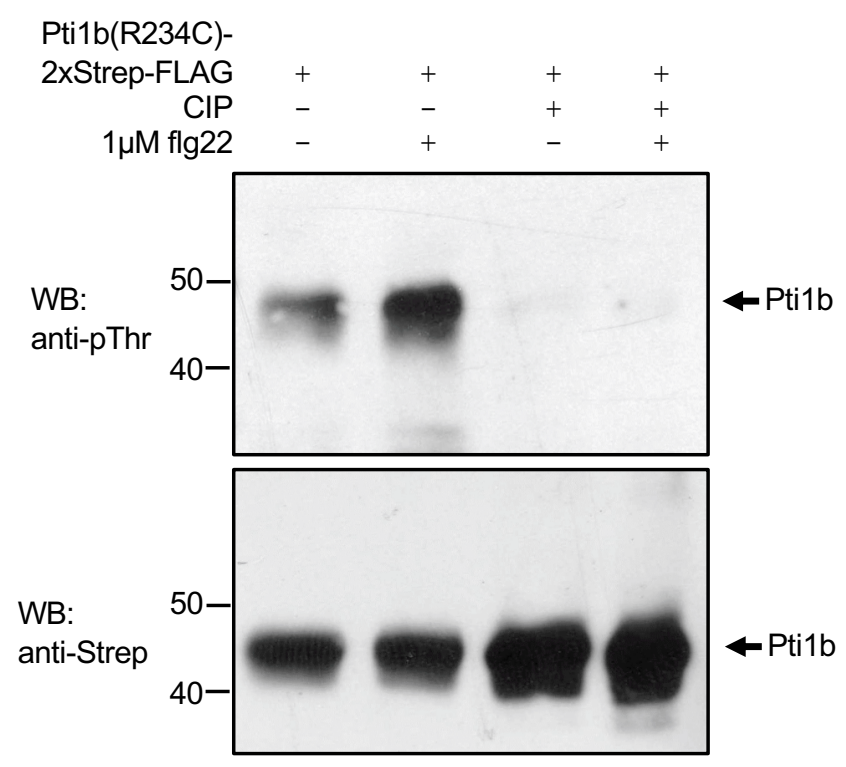

Figure 5. Peptide flg22 induces Pti1b(R234C) phosphorylation on threonine residue(s) in plant cells. Pti1b(R234C)-2xStrep-FLAG was expressed in N. benthamiana leaves using Agrobacterium-mediated transient expression (agroinfiltration). Three days after agroinfiltration, leaf tissue was infiltrated with $1 \mu \mathrm{M}$ flg22 or water as a control. Ten minutes after infiltration the tissue was collected and frozen in liquid nitrogen. Pti1b was purified with Strep-affinity chromatography. Half of the obtained samples were treated with calf intestine phosphatase (CIP) and half were untreated. Pti1b phosphorylation was detected by Western blotting (WB) with antibodies specific to phosphorylated threonine. Protein loading was tested using anti-Strep antibody. This experiment was repeated two times with similar results. 

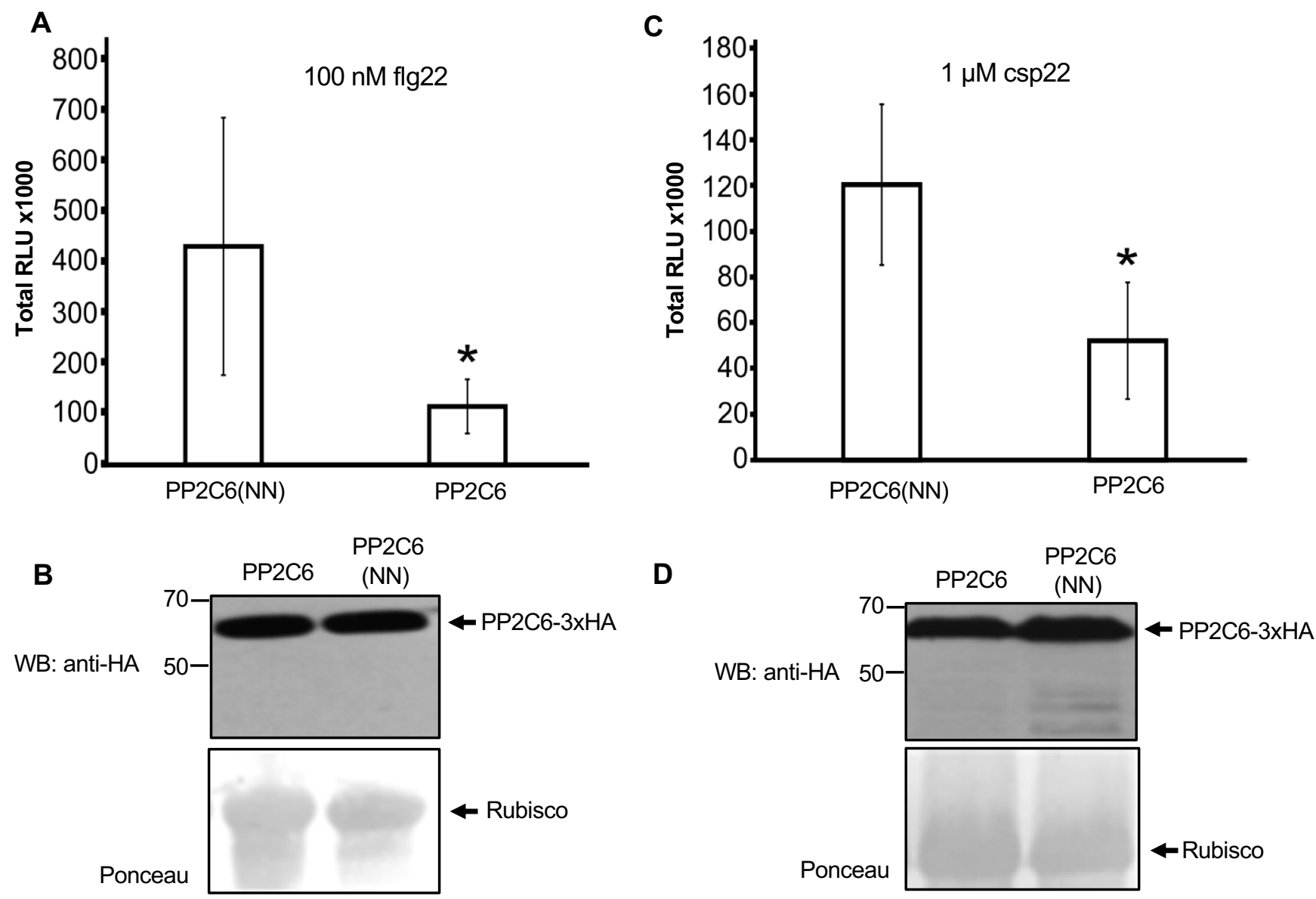

Figure 6. PP2C6 reduces the amount of reactive oxygen species (ROS) produced in $\mathbf{N}$. benthamiana leaves in response to MAMPs. PP2C6 and PP2C6(NN) were expressed in N. benthamiana leaves using agroinfiltration, leaf disks were excised and treated with flg22 or csp22. (A) Total number of relative light units (RLU) detected over 45 minutes after treatment of leaf discs with $100 \mathrm{nM}$ flg22. Bars represent means \pm S.D. $(n=8)$. Asterisk indicates a significant difference based on Welch's test $(p=0.0095)$. (B) PP2C6 variants analyzed in A were detected in leaf tissue by Western blotting (WB) using an anti-HA antibody. (C) Total number of relative light units (RLU) detected over 45 minutes after treatment of leaf discs with $1 \mathrm{uM} \operatorname{csp} 22$. Bars represent means \pm S.D. $(n=8)$. Asterisk indicates significant difference based on Welch's test $(p=0.0009)$. (D) PP2C6 variants analyzed in $\mathrm{C}$ were detected in leaf tissue by Western blotting (WB). To confirm equal loading, the membrane was stained with Ponceau (bottom panels). All the experiments were repeated two times with similar results. 


\section{Supporting Information}

\section{Phosphatase PP2C6 negatively regulates phosphorylation status of Pti1b kinase, a regulator of flagellin-triggered immunity in tomato}

Fabian Giska ${ }^{1}$ and Gregory B. Martin ${ }^{1,2}$

${ }^{1}$ Boyce Thompson Institute for Plant Research, Ithaca, New York 14853, USA

${ }^{2}$ Section of Plant Pathology and Plant-Microbe Biology, School of Integrative Plant Science, Cornell University, Ithaca, New York 14853, USA

\section{Content}

Table S1 Figure S1

Table S2 Figure S2

Table S3 Figure S3

Figure $\mathrm{S4}$

Figure S5

Figure S6 
Table S1. Expression of tomato Pti1a, Pti1b, and PP2C6 genes in response to MAMPs.

Expression of the indicated genes in response to flgll-28 or csp22 treatment of tomato leaves.

RNA sequencing data are from Rosli et al. (2013) Genome Biology 14:R139 and Pombo et al.

(2014) Genome Biology 15:492 and are available from TGFD (http://ted.bti.cornell.edu/cgi-

bin/TFGD/digital/home.cgi, D007 and D010). Transcript abundance was measured as RPKM

(reads per kilobase per exon model per million mapped reads) $6 \mathrm{hr}$ after syringe-infiltration

with $1 \mu \mathrm{M}$ flgll-28, $1 \mu \mathrm{M}$ csp22 or a buffer-only (mock) solution into Rio Grande-prf3 (carrying a

mutation in Prf). Results shown are the ratios between the means of different treatments ( $n=3$

biological replicates), and significant differences were determined by FDR-adjusted $p$-values.

Red shading indicates genes with increased transcript abundance as a result of the treatment, and green indicates no statistically significant change in transcript abundance in response to MAMP treatment.

\begin{tabular}{|c|c|c|c|c|c|}
\hline \multirow{2}{*}{$\begin{array}{c}\text { Gene } \\
\text { name }\end{array}$} & \multirow{2}{*}{ Solyc no. } & \multicolumn{2}{|c|}{$\begin{array}{c}\text { PTI Induction } \\
\text { flgll-28/ } \\
\text { mock }\end{array}$} & \multicolumn{2}{c|}{$\begin{array}{c}\text { PTI Induction } \\
\text { csp22/ } \\
\text { mock }\end{array}$} \\
\cline { 3 - 6 } & & Ratio & FDR & Ratio & FDR \\
\hline Pti1a & Solyc12g098980 & 1.07 & 1 & 1.34 & 0.4878 \\
\hline Pti1b & Solyc05g053230 & 2.68 & $2.42 \mathrm{e}-07$ & 2.56 & $2.31 \mathrm{e}-05$ \\
\hline PP2C6 & Solyc07g066260 & 2.68 & $5.02 \mathrm{e}-07$ & 3.28 & $8.94 \mathrm{e}-08$ \\
\hline
\end{tabular}


Table S2. Primers used in site-directed mutagenesis.

\begin{tabular}{|c|c|}
\hline Primer name & Sequence \\
\hline SIPP2C6(D110N)-F & ATCTTTTGCGGAGTATTTAATGGACACGGTCCCTATG \\
\hline SIPP2C6(D110N)-R & CGTATCACTTCGCGAGCAAAAATTCTCCCAAATG \\
\hline SIPP2C6(D234N)-F & GTTGTTCTAGCAACTAATGGGGTTTGGGATGTACTTTC \\
\hline SIPP2C6(D234N)-R & AAATTCGTTCCTATCAGTTATGCGGTGGTAATAC \\
\hline Pti1b(K96N)-F & GCAGCCATTAACAAGTTAGACTCGAGCAAGC \\
\hline Pti1b(K96N)-R & GCTCGAGTCTAACTTGTTAATGGCTGCAG \\
\hline Pti1b(T233A)-F & GCCTTCACTCTGCACGTGTTCTTGGAACC \\
\hline Pti1b(T233A)-R & GGTTCCAAGAACACGTGCAGAGTGAAGGC \\
\hline Pti1b(T233D)-F & GCCTTCACTCTGACCGTGTTCTTGGAACC \\
\hline Pti1b(T233D)R & GCCTTCACTCTGACCGTGTTCTTGGAACC \\
\hline Pti1b(R234C)-F & CGCCTTCACTCTACATGTGTTCTTGGAACCTTTGGTTATC \\
\hline Pti1b(R234C)-R & TGCTGCCATATCAGGAGCTTGATTTGATAAATCAAAATCC \\
\hline Pti1b(S232A)-F & TGGCAGCACGCCTTCACGCTACACGTGTTCTTGG \\
\hline Pti1b(S232A)-R & TATCAGGAGCTTGATTTGATAAATCAAAATCCGCAATCTTAG \\
\hline Pti1b(S232A)-F in Pti1b(T233A) & TGGCAGCACGCCTTCACGCTGCACGTGTTCTTGGAACCTTTG \\
\hline Pt1ib(S232A)-R in Pti1b(T233a) & TATCAGGAGCTTGATTTGATAAATCAAAATCCGCAATCTTAG \\
\hline Pti1b(R234C)-F in Pti1b(T233A) & CACGCCTTCACTCTGCATGTGTTCTTGGAACCTTTG \\
\hline Pti1b(R234C)-R in Pti1b(T233A) & CTGCCATATCAGGAGCTTGATTTGATAAATCAAAATCCG \\
\hline
\end{tabular}


Table S3. Primers used in molecular cloning.

\begin{tabular}{l|l}
\multicolumn{1}{c|}{ Primer name } & \multicolumn{1}{c}{ Sequence } \\
\hline Pti1b-Ndel (to pET30a+) & ACTGCATATGAGCTGCTTCGGTTGTTGTG \\
\hline Pti1b-Notl (to pET30a+) & ATTCGCGGCCGCCAAGTTTGATGTTTCACTAGGTGCAG \\
\hline Pti1b-Kpnl (to C-nluc-pCambia) & CATCGGTACCATGAGCTGCTTCGGTTGTTGTGAC \\
\hline Pti1b-Sall (to C-nluc-pCambia) & ACCTGTCGACCAAGTTTGATGTTTCACTAGG \\
\hline PP2C6-Kpnl (to C-cluc-pCambia) & ATCCGGTACCATGGGAATTTGTTACTCTTCAAG \\
\hline PP2C6-Sall (to C-cluc-pCambia) & ATCCGTCGACCAACCATTTCCTCCGACTGGCTG \\
\hline GFP-Avrll (to pJLTRBO) & ATCACCTAGGATGGTGAGCAAGGGCGAGGAG \\
\hline GFP-Notl (to pJLTRBO) & ATCAGCGGCCGCTTACTTGTACAGCTCGTCCATGCCG \\
\hline PP2C6-Avrll (to pJLTRBO) & ATCCCCTAGGATGGGAATTTGTTACTCTTCAAGTAAAAGCAAG \\
\hline PP2C6-Notl (to pJLTRBO) & ATCCGCGGCCGCCTAAGCGCTGCACTGAGCAGCG \\
\hline PP2C6-Xbal (to pASK3) & ACCATCTAGATAACGAGGGCAAAAAATGGGAATTGTTACTCTTCAAG \\
\hline
\end{tabular}




\section{Agrobacterium carrying: Pti1b-2xStrep-FLAG}

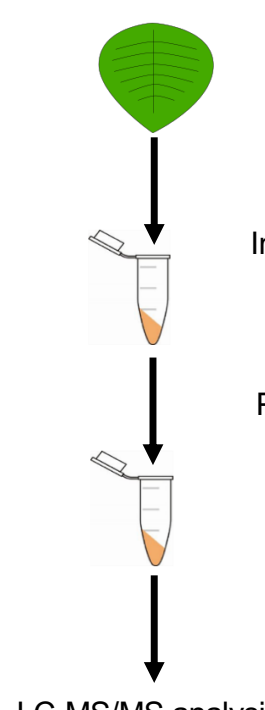

Agrobacterium carrying:

PIP2A-2xStrep-FLAG

(Control)

Agroinfiltration into

N. benthamiana

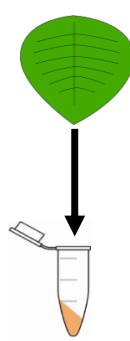

Pull-down purification on

Strep-binding resin

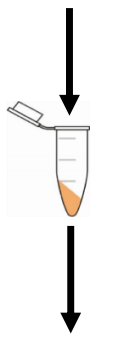

LC-MS/MS analysis

Figure S1. Scheme used to identify plant proteins that co-purify with Pti1b. Pti1b was expressed by agroinfiltration in Nicotiana benthamiana leaves and purified as shown using combined FLAG-tag and Strep-tag affinity chromatography. Transmembrane protein PIP2a was used as a control. After purification, samples were submitted for mass spectrometry analysis to detect proteins that specifically co-purified with Ptilb. 


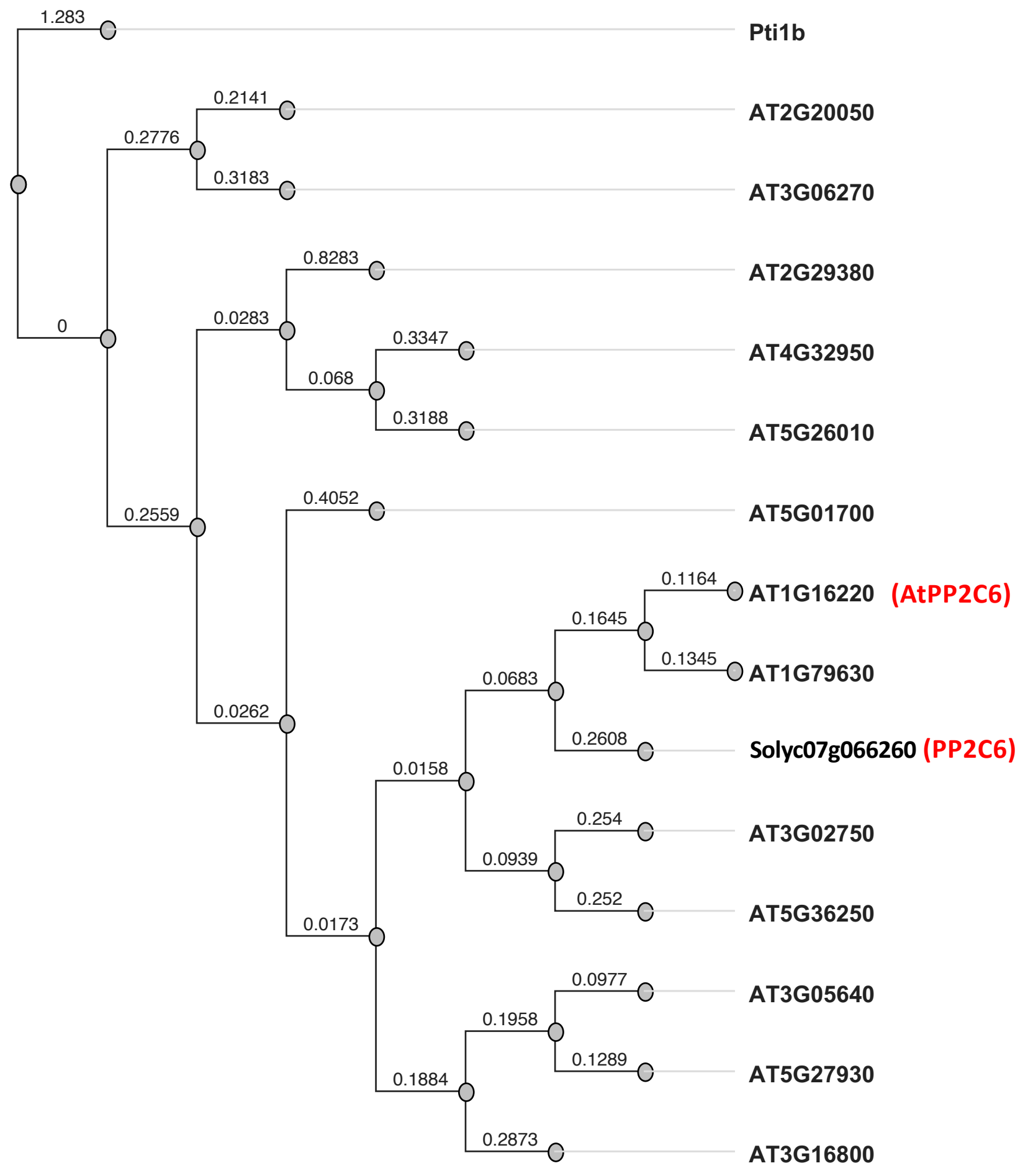

Figure S2. Phylogenetic tree of Arabidopsis phosphatase proteins showing relatedness of AtPP2C6 and PP2C6. The phylogenetic tree was generated with Geneious Tree Builder with the following parameters: alignment type - global alignment, cost matrix - Pam160, genetic distance model - jukes cantor, tree-build method - neighbor-joining, with the amino acid sequence of Pti1b used as the outgroup. Numbers refer to the evolutionary distances between proteins. AtPP2C6 (At1g16220) is the closest homolog of PP2C6 (Solyc07g066260). 

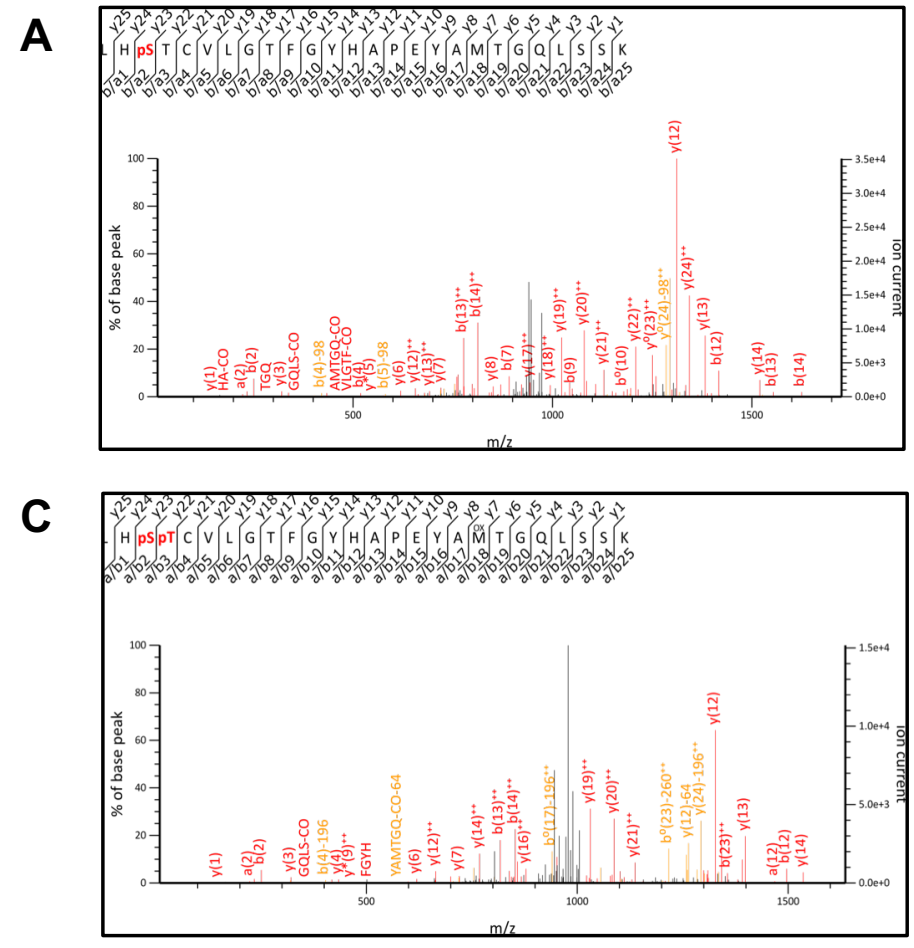

B
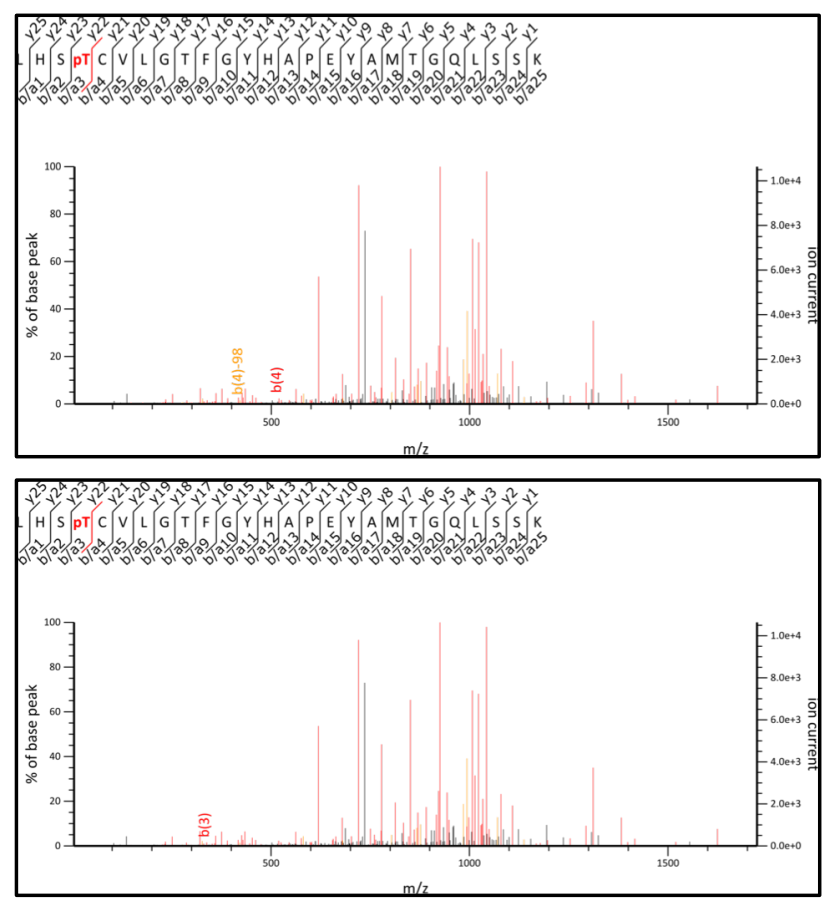

Figure S3. MS/MS spectra of phosphopeptides in Pti1b phosphorylated in vitro. A) Representative spectra for the peptide with phosphorylated serine-232. The ions $b(4), b(5)$ and $y(24)$ shown in orange are the product ions generated by loss of $98 \mathrm{Da}\left(\mathrm{H}_{3} \mathrm{PO}\right)$ from parent ions $\mathrm{b}(4), \mathrm{b}(5)$ and $\mathrm{y}(24)$ shown in red. B) Upper panel: Representative spectra for peptide with phosphorylated threonine-233. The ion b(4) shown in orange is a product of ion generated by loss of $98 \mathrm{Da}\left(\mathrm{H}_{3} \mathrm{PO}\right)$ from parent ion $\mathrm{b}(4)$ shown in red. Bottom panel - the product ion of parent ion $b(3)$ was not detected which supports the conclusion that the phosphothreonine233 but not phosphoserine-S232 is a source of $\mathrm{H}_{3} \mathrm{PO} 4$ group in ion $\mathrm{b}(4)$ from upper panel. $\mathrm{C}$ ) The ions $\mathrm{b}(4)$, $b(5)$ and $y(24)$ in orange are the product ions generated by loss of $196 \mathrm{Da}$ (two $\mathrm{H}_{3} \mathrm{PO} 4$ group) from parent ions $b(4), b(5)$ and $y(24)$ in red. 
A Pti1b

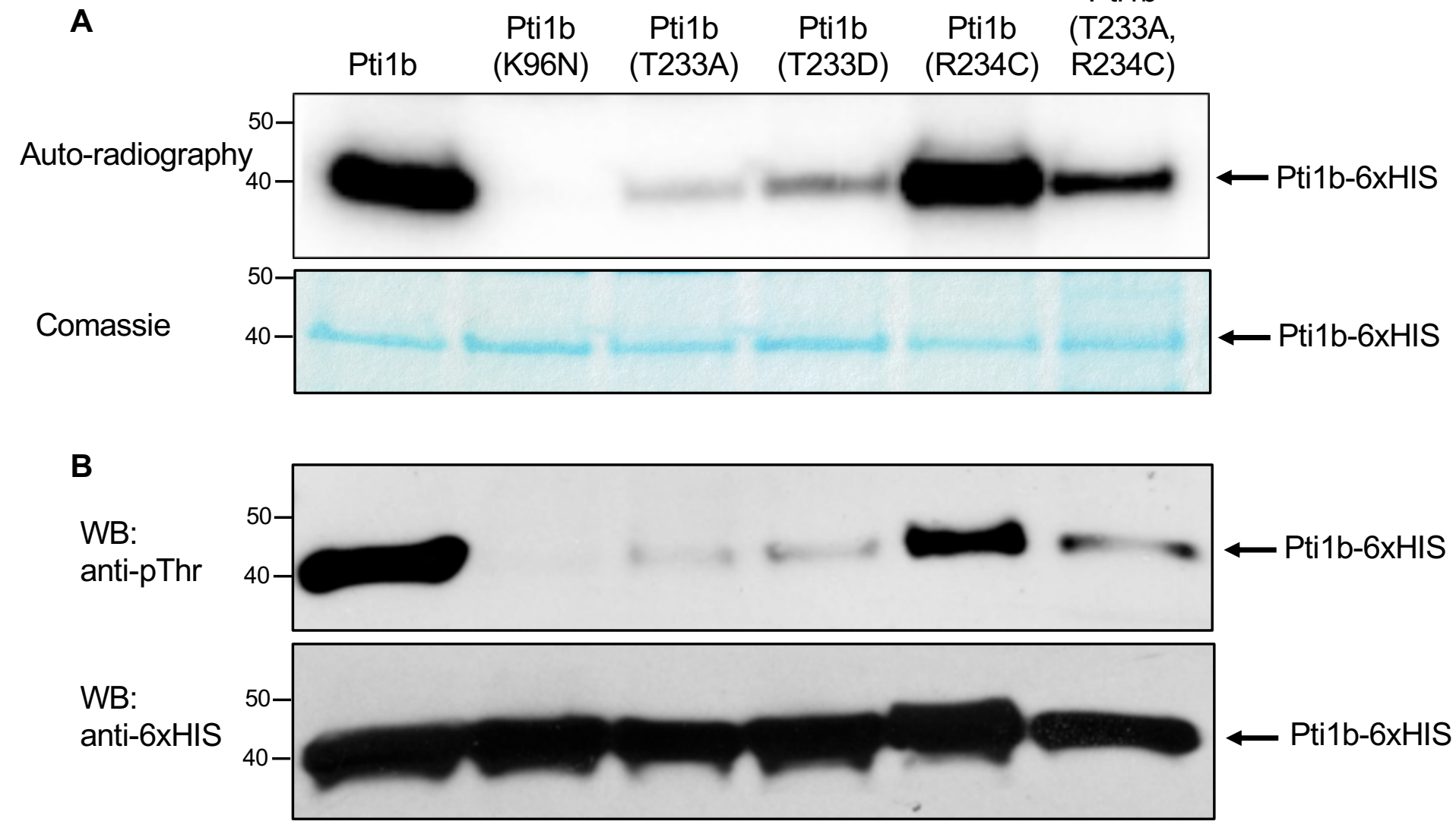

Figure S4. Testing the specificity of the anti-phosphothreonine antibodies used in experiment

showed in Figure 5. A) Upper panel: autophosphorylation of different Pti1b isoforms was examined with autoradiography; lower panel: total amount of protein loaded was checked with Coomassie blue staining. B) Upper panel: autophosphorylation of different Pti1b isoforms was detected using Western blotting with anti-phosphothreonine antibodies; lower panel: the amount of loaded protein was tested with anti-6xHIS antibodies. The experiment was repeated twice with similar results. 


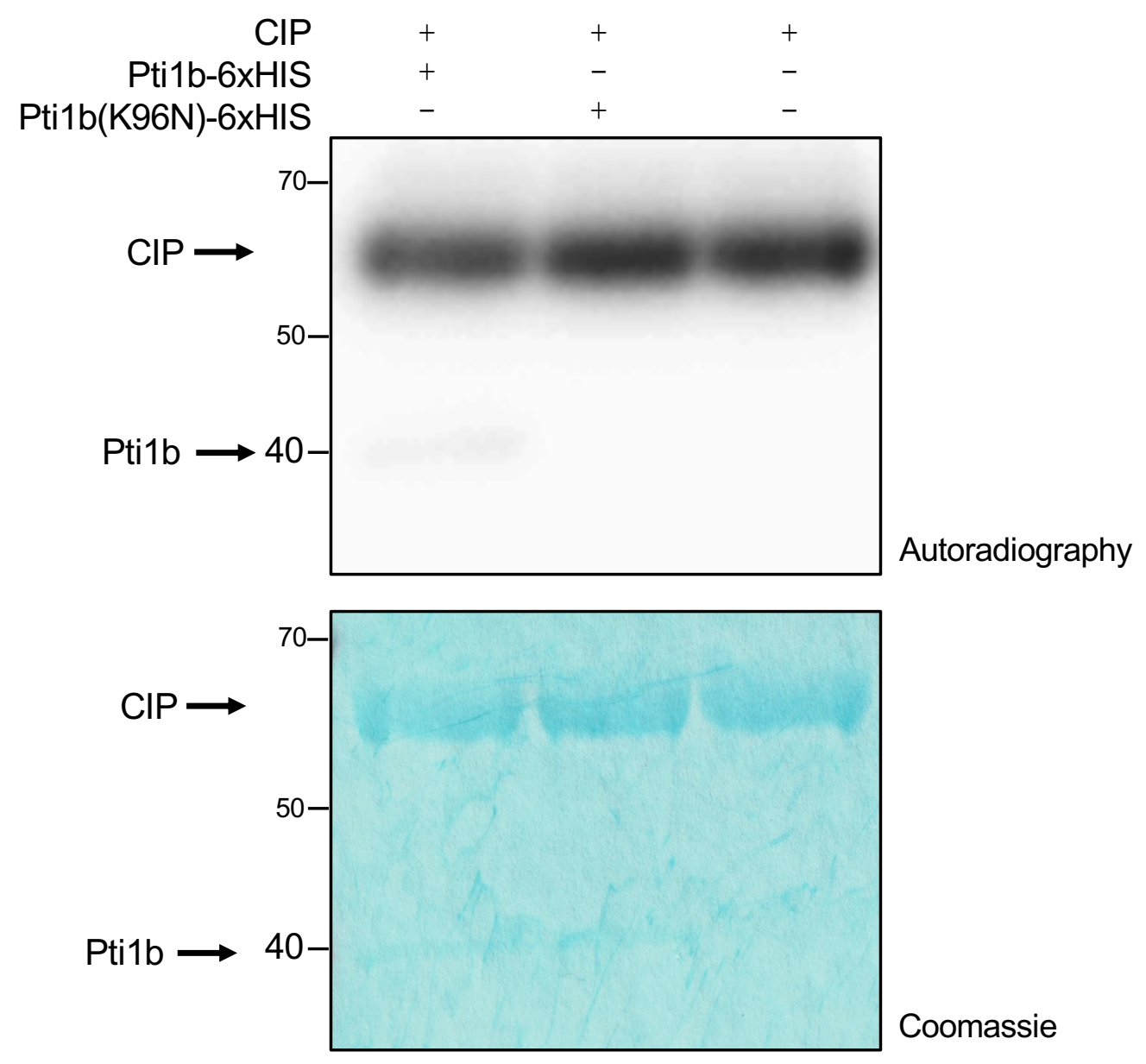

Figure S5. The phosphorylation status of CIP is not dependent on kinase activity of Pti1b. CIP phosphatase was incubated alone or in the presence of Pti1b or an inactive variant, Pti1b(K96N). The phosphorylation status of the proteins was examined with autoradiography (upper panel). The amount of protein loaded was checked with Coomassie staining (lower panel). 


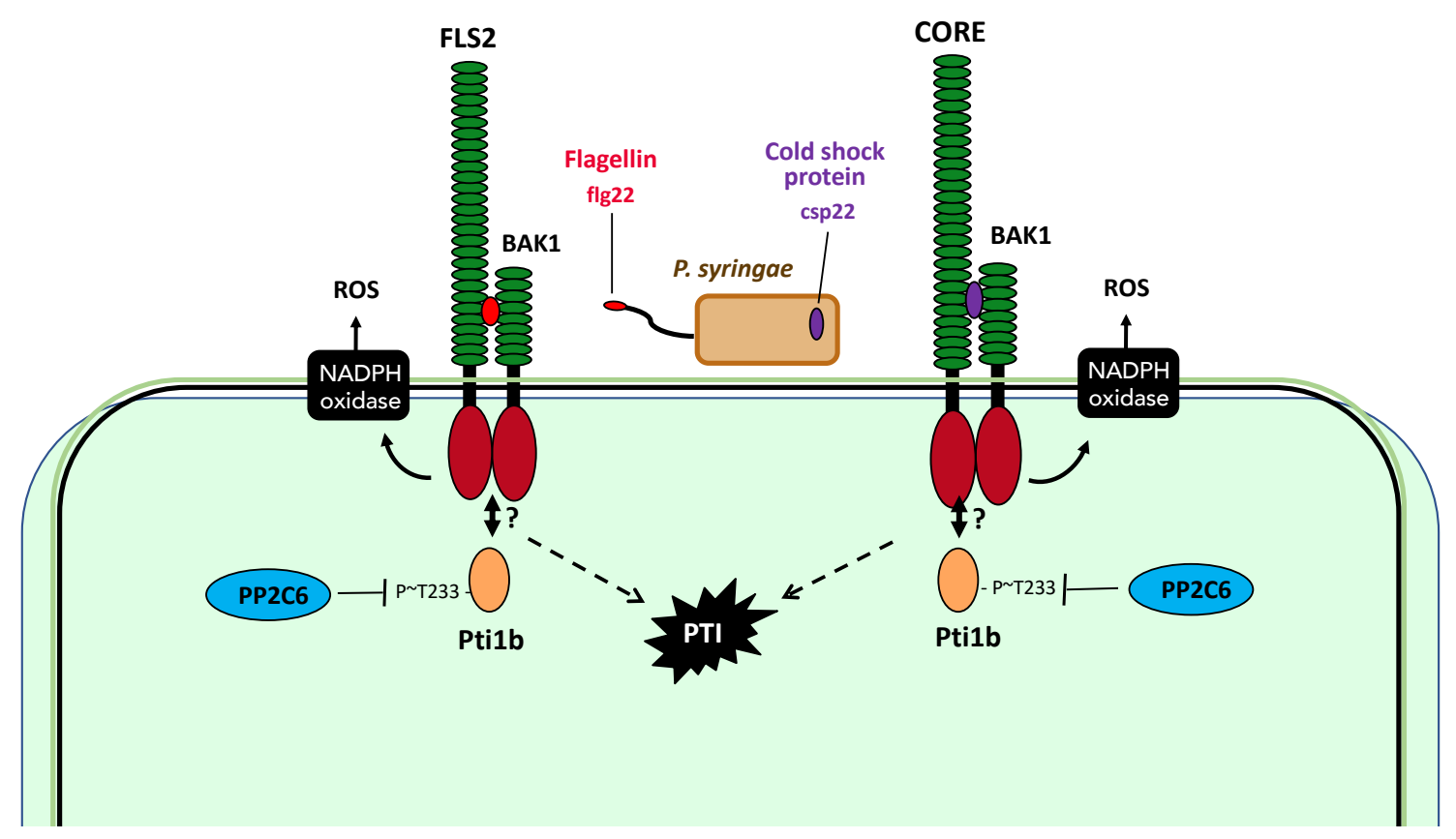

Figure S6. A model for negative regulation of Pti1b kinase by the PP2C6 phosphatase. The model shows that in the presence of a microbe expressing flg22 or csp22, the FLS2 and CORE receptors, respectively, directly or indirectly activate the Pti1b kinase by increasing phosphorylation of threonine-233 in Pti1b which activates downstream pattern-triggered immunity (PTI) responses. PP2C6 dephosphorylates threonine-233 in Pti1b thereby negatively regulating Pti1b-mediated PTI responses. See text for further discussion of the model. 